Portland State University

PDXScholar

\title{
Bi-objective Optimization for Battery Electric Bus Deployment Considering Cost and Environmental Equity
}

Xiaoyue Cathy Liu

The University of Utah

Yirong Zhou

The University of Utah

Ran Wei

University of California, Riverside

Aaron Golub

Portland State University, agolub@pdx.edu

Devin Macarthur

Portland State University

Follow this and additional works at: https://pdxscholar.library.pdx.edu/trec_reports

Part of the Transportation Commons, Urban Studies Commons, and the Urban Studies and Planning Commons

Let us know how access to this document benefits you.

\section{Recommended Citation}

Liu, X., Zhou, Y., Wei, R., Golub, A. and Macarthur, D. Bi-objective Optimization for Battery Electric Bus Deployment Considering Cost and Environmental Equity. NITC-RR- 1222. Portland, OR: Transportation Research and Education Center (TREC), 2021.https://dx.doi.org/10.15760/trec.256

This Report is brought to you for free and open access. It has been accepted for inclusion in TREC Final Reports by an authorized administrator of PDXScholar. Please contact us if we can make this document more accessible: pdxscholar@pdx.edu. 


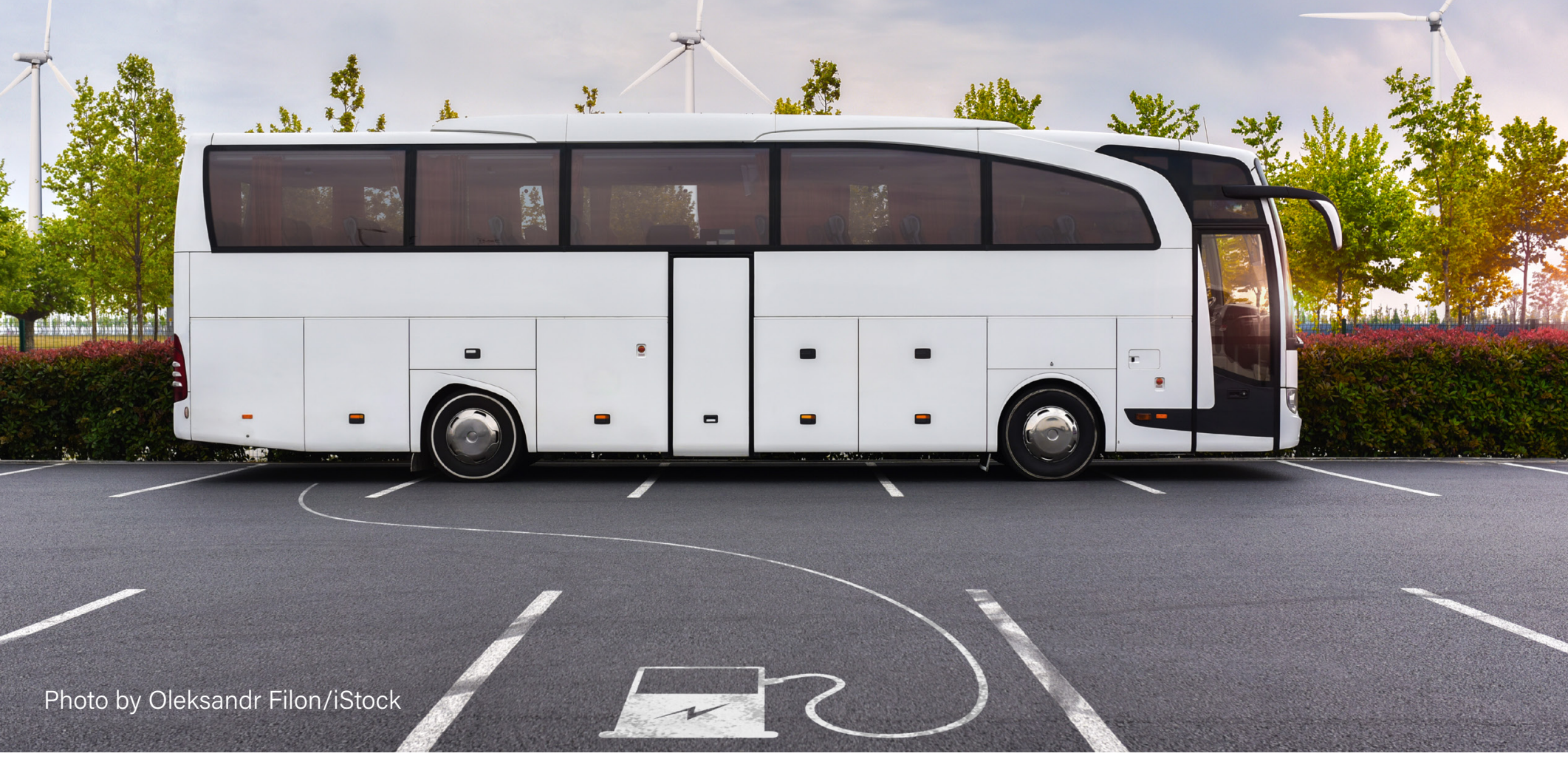

\section{Bi-objective Optimization for Battery Electric Bus Deployment Considering Cost and Environmental Equity}

Xiaoyue Cathy Liu, Ph.D

Yirong Zhou

Ran Wei, Ph.D

Aaron Golub, Ph.D.

Devin Macarthur

U. 


\title{
Bi-objective Optimization for Battery Electric Bus Deployment Considering Cost and Environmental Equity
}

\author{
Final Report
}

NITC-RR-1222

\author{
by \\ Xiaoyue Cathy Liu (PI) \\ Yirong Zhou \\ University of Utah \\ Ran Wei (co-PI) \\ University of California, Riverside \\ Aaron Golub (co-PI) \\ Devin Macarthur \\ Portland State University
}

for

National Institute for Transportation and Communities (NITC)

P.O. Box 751

Portland, OR 97207
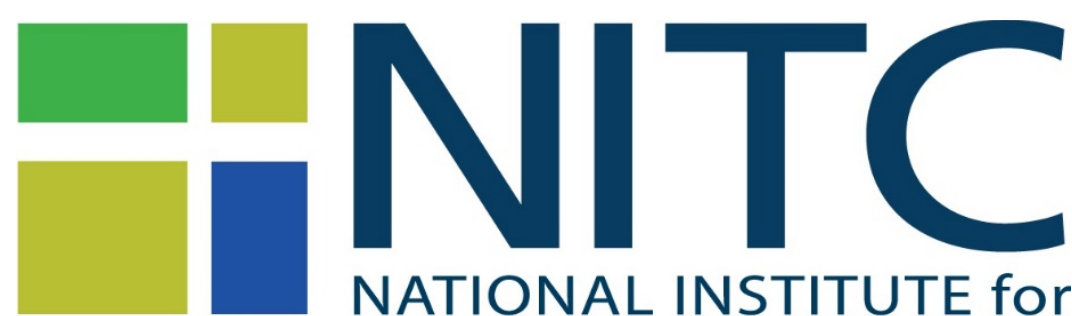

NATIONAL INSTITUTE for TRANSPORTATION and COMMUNITIES

March 2021 


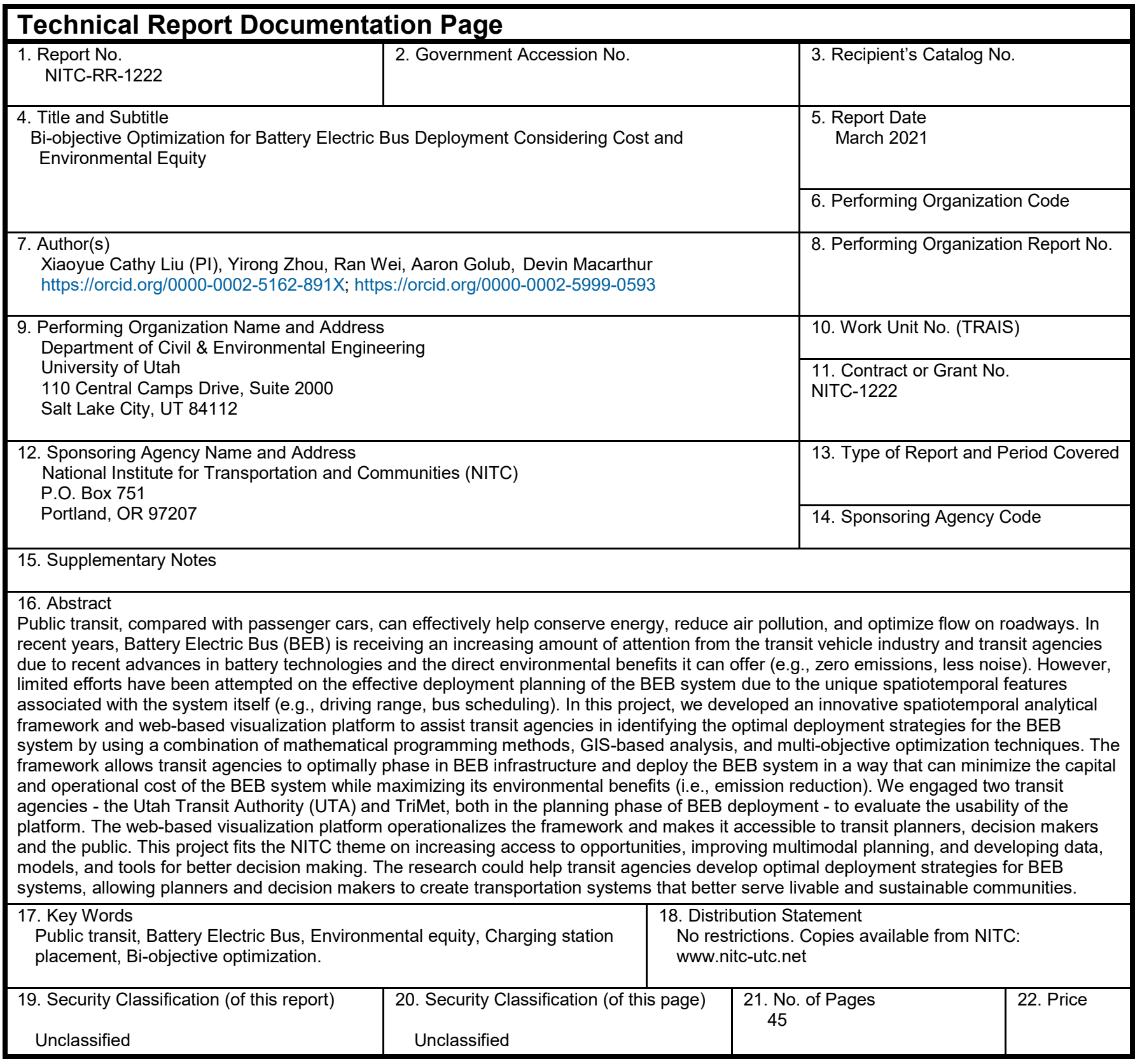




\section{ACKNOWLEDGEMENTS}

This project was funded by the National Institute for Transportation and Communities (NITC; grant number 1222) a U.S. DOT University Transportation Center. The project also benefitted from matches from the University of Utah, Portland State University, and the University of California at Riverside. Furthermore, we acknowledge and thank the anonymous peer reviewers who provided helpful insights and corrections to the report, which is published in the IEEE Transactions on Intelligent Transportation Systems (Zhou et al., 2020).

\section{DISCLAIMER}

The contents of this report reflect the views of the authors, who are solely responsible for the facts and the accuracy of the material and information presented herein. This document is disseminated under the sponsorship of the U.S. Department of Transportation University Transportation Centers Program in the interest of information exchange. The U.S. Government assumes no liability for the contents or use thereof. The contents do not necessarily reflect the official views of the U.S. Government. This report does not constitute a standard, specification, or regulation.

\section{RECOMMENDED CITATION}

Liu, X., Zhou, Y., Wei, R., Golub, A. and Macarthur, D. Bi-objective Optimization for Battery Electric Bus Deployment Considering Cost and Environmental Equity. NITC-RR1222. Portland, OR: Transportation Research and Education Center (TREC), 2021. 


\section{TABLE OF CONTENTS}

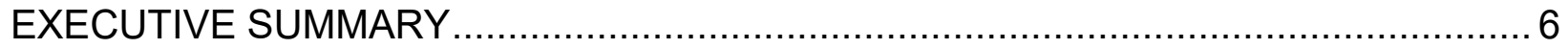

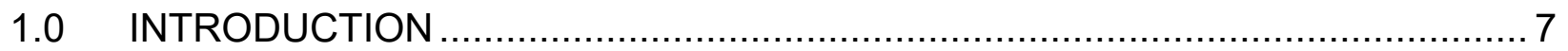

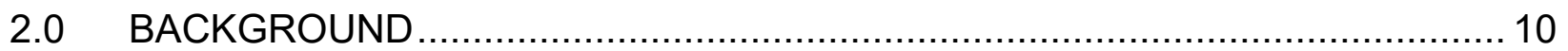

2.1 DIESEL OR CNG BUSES-RELATED RESEARCH $\ldots \ldots \ldots \ldots \ldots \ldots \ldots \ldots \ldots \ldots \ldots \ldots \ldots$

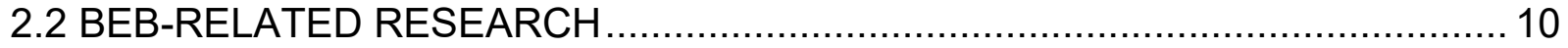

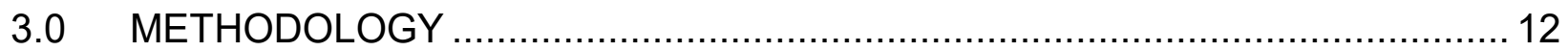

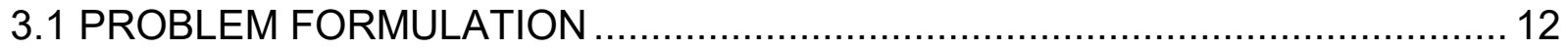

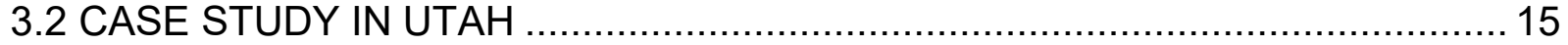

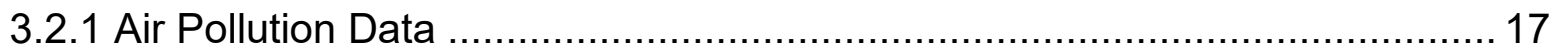

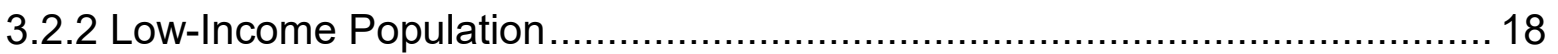

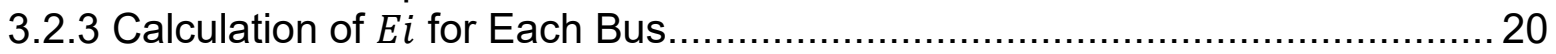

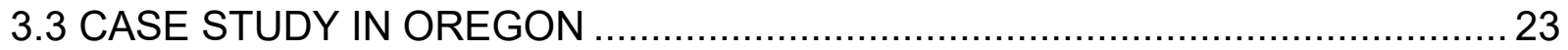

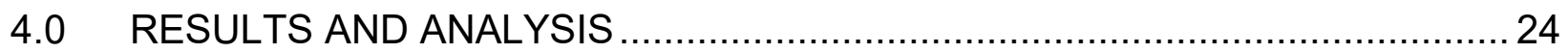

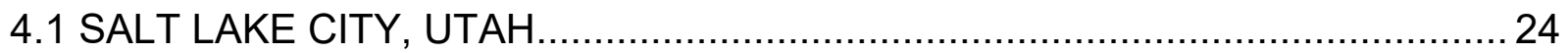

4.1.1 Trade-off between Costs and Environmental Equity ............................ 24

4.1.2 Examples of Deployment Plans ...................................................... 24

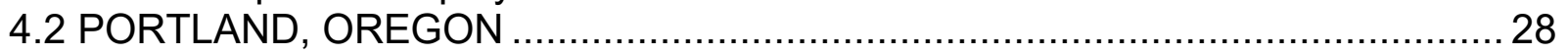

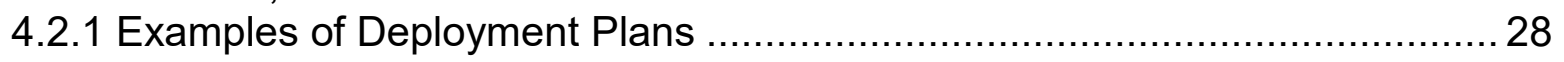

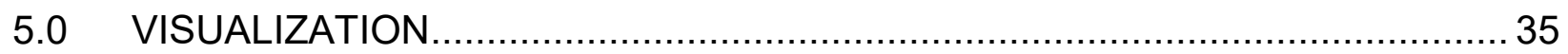

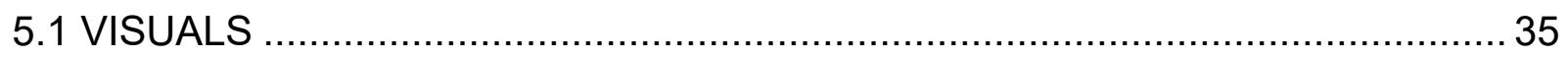

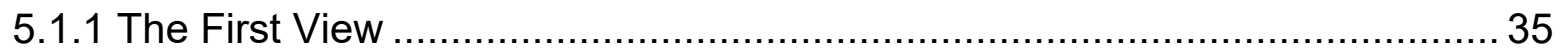

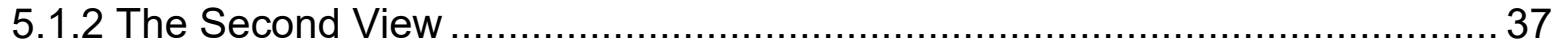

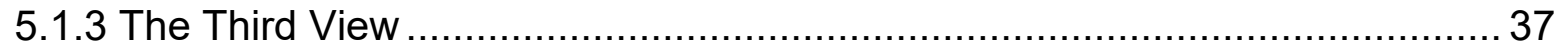

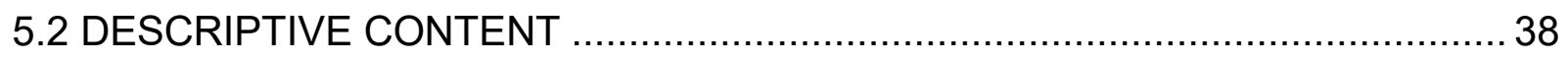

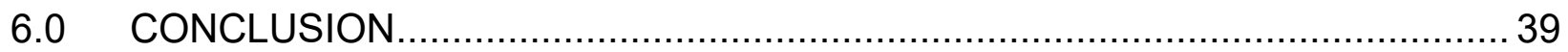

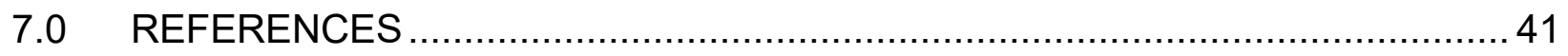




\section{LIST OF FIGURES}

Figure 3.1: Study Area 17

Figure 3.2: Sample Screenshot of PurpleAir Sensor Distribution in the State of Utah on $04 / 08 / 2020$ 18

Figure 3.3: Distribution of Low-Income Populations .................................................. 19

Figure 3.4: PM2.5 Concentration Delineated by TAZ for Utah ................................ 20

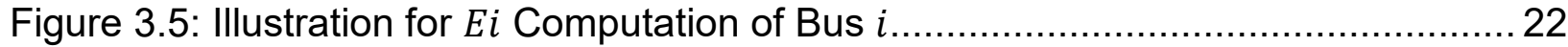

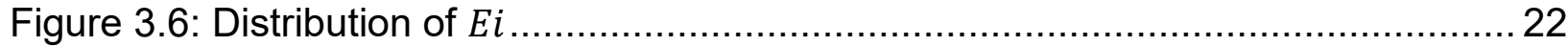

Figure 4.1: Trade-off Curve between Cost and Environmental Equity ........................2 24

Figure 4.2: BEB Deployment Plan when Budget is set at $\$ 25$ million ........................26

Figure 4.3: BEB Deployment Plan when Budget is set at $\$ 60$ million ....................... 27

Figure 4.4: BEB Deployment Plan when Budget is set at $\$ 120$ million .......................2 28

Figure 4.5: Maximum Environmental Equity with Five BEB Replacements .................. 29

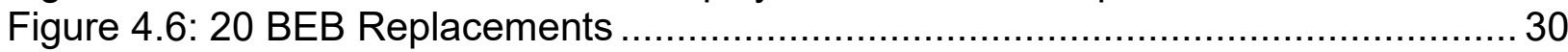

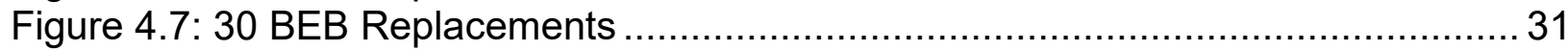

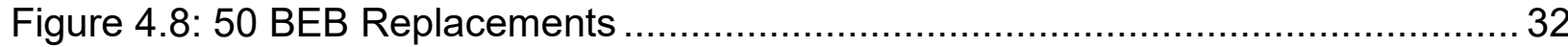

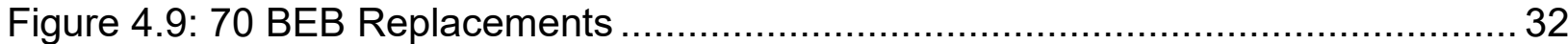

Figure 4.10: Selected Lines 71 - 60th and 72 - Killingsworth/82 ${ }^{\text {nd }}$ Ave....................... 33

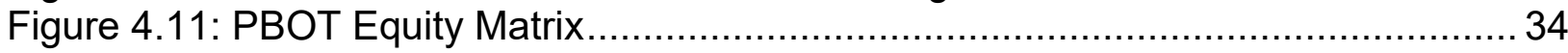

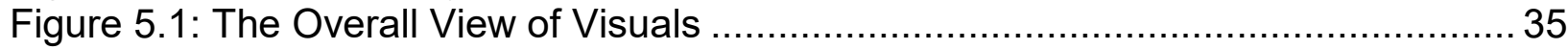

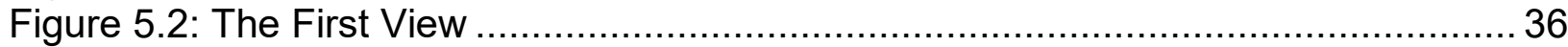

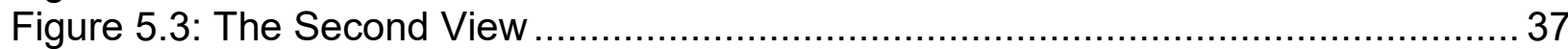

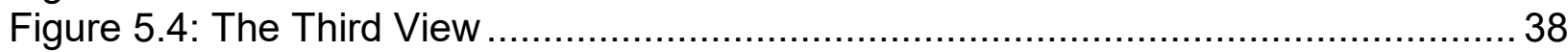

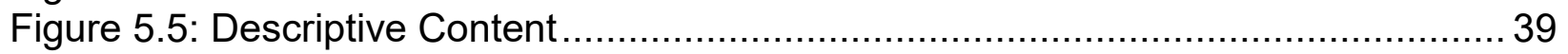

\section{LIST OF TABLES}

Table 4.1: TriMet's Plan to Purchase 70 BEBs by 2022 .................................................. 29 


\section{EXECUTIVE SUMMARY}

Public transit, compared with passenger cars, can effectively help conserve energy, reduce air pollution, and optimize flow on roadways. In recent years, Battery Electric Bus (BEB) is receiving an increasing amount of attention from the transit vehicle industry and transit agencies due to recent advances in battery technologies and the direct environmental benefits it can offer (e.g., zero emission, less noise). However, limited efforts have been attempted on the effective deployment planning of the BEB system due to the unique spatiotemporal features associated with the system itself (e.g., driving range, bus scheduling). In this project, we developed an innovative spatiotemporal analytical framework and web-based visualization platform to assist transit agencies in identifying the optimal deployment strategies for the BEB system by using a combination of mathematical programming methods, GIS-based analysis, and multi-objective optimization techniques. The framework allows transit agencies to optimally phase in BEB infrastructure and deploy the BEB system in a way that can minimize the capital and operational cost of the BEB system while maximizing its environmental benefits (i.e., emission reduction). We engaged two transit agencies - the Utah Transit Authority (UTA) and TriMet, both in the planning phase of BEB deployment - to evaluate the usability of the model. The web-based visualization platform operationalizes the framework and makes it accessible to transit planners, decision makers and the public. This project fits the NITC theme on increasing access to opportunities, improving multimodal planning, and developing data, models, and tools for better decision making. The research could help transit agencies develop optimal deployment strategies for BEB systems, allowing planners and decision makers to create transportation systems that better serve livable and sustainable communities. 


\subsection{INTRODUCTION}

Public transit systems are key to moving mass populations efficiently and in a way that is environmentally friendly. Compared with passenger cars, public transit can effectively help conserve energy, reduce air pollution, and optimize traffic flow on roadways. Motivated by the advancement of battery technology and the increasing need for a cleaner source of energy, Battery Electric Bus (BEB) is receiving a growing amount of attention from the transit vehicle industry and transit agencies (Glotz-Richter and Koch, 2016; Li, 2016; Lajunen, 2014). Automotive companies like Proterra, New Flyers, and BYD have been continuing their investments in BEB-related technology. Over the past eight to 10 years, companies as such have built mature product lines of $B E B$ and associated charging infrastructures. This combined with reduced battery price has made the large-scale commercial deployment of BEB possible. Correspondingly, many transit agencies have made long-term and/or short-term plans for replacing their existing fleet with BEB. The Los Angeles County Metropolitan Transportation Authority (LA Metro) announced in July 2017 that its transit fleet will complete electrification by 2030, requiring at least 2,300 BEBs (Miller et al., 2020). MTA New York City Transit began to test five New Flyer BEBs across its system in February 2018 and similar tests have been piloted in Boston, Portland, Seattle, and Salt Lake City (Miller et al., 2020). The transit industry is rapidly transitioning to battery-electric fleets due to the direct environmental and financial benefits they could offer, such as zero emissions, less noise, and lower maintenance costs (Filippo et al., 2018; Xylia and Silveira, 2014). Meanwhile, the transit system is what social functions depend highly upon, especially in areas where disadvantaged populations are transit-dependent and tend to be the socioeconomic groups that are particularly vulnerable to air pollution (Fayyaz et al., 2017; Pratt et al., 2015). Full electrification could potentially improve environmental equity significantly.

Current research on BEBs has been focusing on energy consumption analysis (ElTaweel et al., 2020; Sinhubera et al., 2012; Tzeng et al., 2005); charging infrastructures placement (He et al., 2013; Wang et al., 2017; He et al., 2015; Xylia et al., 2017; Liu et al., 2020; Sebastiani et al., 2016); optimizing charging schemes (Liu et al., 2020; Sebastiani et al., 2016; Yang et al., 2018; Qin et al., 2016); fleet replacement (Pelletier et al., 2019; Wei et al., 2018); and cost-benefits analysis (Lajunen, 2014; McKenzie and Durango-Cohen, 2012). Most of the work deals with small-scale systems where only simplified situations or assumptions are considered, such as a single bus route, fixed number of charging stations, or limited charging times. Very few efforts have been attempted on the effective deployment planning of a large-scale BEB system with empirical data. Also, many studies used simulated data to validate their models when empirical data is unavailable, which greatly hindered the possibility of model adoption by the transit agencies. On top of that, cost has always been a dominant focus when optimizing the BEB deployment, yet important goals such as environmental equity are often neglected.

This research develops a bi-objective spatiotemporal optimization model for the strategic deployment of BEB. The first objective is to minimize the cost of purchasing 
BEB and installing both on-route and in-depot charging stations while maintaining current bus schedules. The other objective is to maximize environmental equity by incorporating the disadvantaged population in the decision-making process. Research on social vulnerability found that low socioeconomic status (SES) groups often experience a higher concentration of air pollutants due to the low value of lands and the closeness to income-earning opportunities (Hajat et al., 2015). Case studies have been conducted across the world in many major cities. For example, Bell et al. (2012) studied environmental inequality with regard to airborne particulate matter exposure in the United States. They used daily air pollution measures obtained for seven consecutive years (2000-2006) to match the U.S. census tracts from the 2000 Census. They drew a similar conclusion that persons with lower SES had higher estimated exposure. Other research conducted by Hajat et al. (2013) and Fecht et al. (2015) also concluded with similar results. However, exceptions exist such as New York City, where higher SES groups suffer more from the air pollution. These exceptions are also pointed out in Hajat et al. (2013) and Fecht et al. (2015). Hajat et al. gave a possible explanation that the scenic views and easy access to urban amenities attract high SES individuals to reside close to busy roadways. Therefore, one of our fundamental assumptions is that lowincome groups tend to suffer more from air pollution. To this end, when considering optimal BEB deployment, environmental equity is quantified via disadvantaged populations weighted by the air pollutant concentration. The deployment is to ensure that the places where low-income populations suffer the most from unhealthy air quality could receive priority.

The developed bi-objective spatiotemporal optimization model along with the results are integrated via a unifying interactive visualization platform to support querying, navigating, and exploring various BEB deployment scenarios. The knowledge discovery is spatiotemporal in nature, and we focus on effective visualization designs that are interactive, intuitive, and informative. Our web-based visualization platform utilized the transit network of the Utah Transit Authority (UTA) to demonstrate our proposed method. The platform allows users to interactively explore the designated buses to be replaced with $\mathrm{BEBs}$ with their customized inputs, the siting of corresponding charging stations, as well as the impacts of various BEB deployment strategies in terms of cost and environmental/social benefits.

In sum, the main contributions of our project are threefold:

- We developed a bi-objective spatiotemporal optimization model for the strategic deployment of BEBs to minimize the cost of purchasing BEBs, on-route and in-depot charging stations, and to maximize environmental equity for disadvantaged populations. The optimization considers the unique constraints imposed by BEB operations in a spatiotemporal fashion.

- We used empirical data to offer a potential framework that can be adopted or expanded by transit agencies to optimally deploy BEBs by accommodating multiple goals and objectives that the transit agencies set forth. 
- We built a unifying interactive visualization platform to support querying, navigating, and exploring various BEB deployment scenarios. Users are able to explore multiple BEB deployment scenarios with user-specified inputs. The platform will then output corresponding parameters for each scenario, along with the BEB deployment plan (i.e., trajectories of buses to the replaced, charging station location).

The remainder of this report is organized as follows. Literature related to BEB deployment and potential environmental benefits brought by vehicle electrification is discussed in the Background section. The Methodology section presents the biobjective optimization model and further discusses the definition of variables, parameters, and constraints with examples. The Application section is divided into two subsections, Data Source and Results. In the Data Source section, details on the source and structure of air quality data, sociodemographic data, and transit network data in Salt Lake City, Utah, and Portland, Oregon, are presented. The Results section demonstrates the calculation for all parameters and various deployment plans under different budgets. The Visualization section presents the interactive platform we developed to allow users to visually explore the modeling results. In the Conclusion section, the contribution of this project is summarized and the potential future work is discussed. 


\subsection{BACKGROUND}

\subsection{DIESEL OR CNG BUSES-RELATED RESEARCH}

The advancement in battery technology (Li, 2016; Lajunen, 2014) makes large-scale adoption of BEBs a feasible solution to sustainable commuting. However, when compared to traditional diesel or Compressed Natural Gas (CNG) buses, BEB-related research faces new challenges due to the limitations on battery capacity, high capital cost, constrained driving range, and needs for charging infrastructures support, etc. Previous research on diesel or CNG buses mainly focused on the designs of transit service (e.g., coverage area, scheduling) (Spasovic et al., 1993; Ibeas et al., 2010) and/or bus itself (e.g., fleet size, occupancy) (dell'Olio et al., 2010). Research as such is still of significance to the BEB system because BEBs and diesel or CNG buses serve the same functionality, such as reducing traffic congestion, increasing access to employment opportunities, and lowering transportation costs for the public. However, BEBs have additional constraints that need to be considered for deployment. For example, Spasovic et al. (1993) presented a framework to optimize transit service coverage. In the framework, they tried to find the optimal length of transit routes that extend radially from the central business district (CBD) into low-density suburbs as well as route spacing, headway, fare, etc. For BEBs, other aspects such as potential charging locations, charging time, and driving range also need to be examined carefully on top of those aforementioned factors. The same considerations have to be given when BEBs are the target (Ibeas et al., 2010). The authors attempted to optimize bus stop spacing in urban areas using a bi-level optimization model. In this case, a natural constraint for BEBs would be the constraint forcing the spacing between two potential charging sites to be within the driving range. In summary, solving BEB-related problems is similar to traditional transit problems, yet it requires additional considerations of the unique features that BEBs possess.

\subsection{BEB-RELATED RESEARCH}

Recently, a lot of research has been conducted on BEB-related issues. A myriad of studies has examined the charging station placements for private or alternative fuel vehicles associated with driving behavior and battery usage analysis (He et al., 2013; He et al., 2015). Xylia et al. (2018) presents a model to optimally deploy electric vehicle charging stations at selected bus stops to minimize the total cost of building charging infrastructures. The effectiveness of the model was demonstrated using simulated data. Similar research such as Xylia et al. (2017) also presents a siting plan of charging stations to minimize capital investment. Fleet replacement has been studied in Pelletier et al. (2019). However, these approaches either made simplified assumptions, only considering the cost for optimization, or did not consider the unique spatiotemporal characteristics associated with the BEB system. For the BEB system, due to the battery capacity, it has mileage constraints and requires periodic charging via either on-route and/or in-depot charging. In any transit system, it maintains specific transit operation 
routes and schedules. The adoption of BEBs should thus integrate into current routes and schedules seamlessly (given the BEB's constraints) to ensure a smooth transition from diesel or CNG buses. This means that the selection of buses and routes for BEB replacement should consider its spatiotemporal characteristics, such as available time window for on-route charging, conflicting charging demand, and bus trajectories. More importantly, all these studies assume the replacement of the entire fleet with BEB, while in reality due to budget and stage-wise planning, transit agencies often prefer to replace parts of the fleet at different phases. For instance, the problem introduced in Xylia and Silveira (2014) did not consider actual bus schedules. Wang et al. (2017) only considered a limited number of selected bus routes. The replacement plan in Pelletier et al. (2019) did not consider the location requirement of on-route and in-depot charging stations. Wei et al. (2018) allowed for the partial replacement of the bus fleet but did not consider factors other than cost.

Another stream of research on BEBs explored the environmental benefit gained through the transition. Life-cycle assessment of greenhouse gas emissions was assessed in McKenzie et al. (2012), Rupp et al. (2020), Nordelöf et al. (2019), Islam and Lownes (2019), and Dreier et al. (2018) as well as air pollutant emissions (Liberto et al., 2018). A new methodology was studied in Rupp et al. (2020) to optimize charging time as a function of $\mathrm{CO} 2$ emissions and the cost of electricity. The life-cycle environmental impacts of city buses were assessed in Nordelöf et al. (2019) for buses with different levels of electrification, charging options, and types of fuels for combustion engines. On top of the studies related to greenhouse gas emission, a case study in Roma, Italy, (Liberto et al., 2018) explored the changes in energy demand and resulting greenhouse gas and air pollutant emissions.

The aforementioned studies have been focusing on either cost or environmental benefits associated with BEB deployment. However, public transit planning needs to consider social equity, particularly since the majority of transit dependents are less privileged populations (Garrett and Taylor, 1999). Those populations tend to suffer the most from air pollution as they oftentimes reside in areas with a high concentration of air pollutants. By replacing BEB with diesel or CNG buses in those polluted neighborhoods, it further improves the potential environmental and social equity. 


\subsection{METHODOLOGY}

\subsection{PROBLEM FORMULATION}

The bi-objective model relies upon the following notation:

Indices:

$i=$ index of buses (complete set $I$ )

$m=$ index of on - route charging stations (complete set $M$ )

$n=$ index of in - depot charging stations (complete set $N$ )

$s=$ index of bus terminal sequence

$t=$ index of time sequence

Parameters:

$E_{i}=$ environmetal equity reached by replacing bus $i$

$C_{m}^{O}=$ cost of building one on - route charging station at $m$

$C_{n}^{I}=$ cost of building one in - depot charging station at $n$

$C^{B}=$ cost of purchasing one $B E B$

$p^{O}=$ number of BEBs that one on

- route charging station can charge simultaneously

$p^{I}=$ number of BEBs that one in

- depot charging station can charge simultaneously

$l_{i, s-1, s}=$ route distance between terminals $s$ and $s-1$ for bus $i$

$R=$ driving range for $B E B$ without charging

$T D_{i}=$ total driving distance for bus $i$ in one day

$\alpha_{m}=$ set of bus terminal sequence at $m$

$\beta_{m t}=$ set of sequences for bus arriving at $m$ and time $t$

$\gamma_{n}=$ set of buses charged at n overnight

Decision Variables:

$X_{i s}= \begin{cases}1 & \text { bus } i \text { is charged at } s \\ 0 & \text { o.w. }\end{cases}$ 
$Y_{m}^{O}=$ number of on - route charging stations built at $m$

$Y_{n}^{I}=$ number of in - depot charging stations built at $n$

$Z_{i}= \begin{cases}1 & \text { bus } i \text { is replaced with } B E B \\ 0 & \text { o.w. }\end{cases}$

$D_{i s}=$ distance traveled by bus $i$ at sequence $s$

For a typical weekday, one diesel or CNG bus, indexed by $i$, runs through a sequence of terminals, indexed by $s$. Among all the sequences, those that satisfy certain constraints can be perceived as potential sites for building on-route charging stations, which are indexed by $m$. Garages for overnight charging are indexed by $n$. The arrival time of bus $i$ at any terminal is indexed by $t$.

Take a particular bus $i$ as an example. Bus $i$ travels from terminal $w_{1}$ at time $t_{1}$ through terminal $w_{2}$ at time $t_{2}$ to terminal $w_{3}$ at time $t_{3}$. Then bus $i$ arrives at terminal $w_{4}$ at time $t_{4}$ and goes back to terminal $w_{1}$ at time $t_{5}$. Bus $i$ repeats the trip five times a day. The environmental equity it reached is labeled $E_{i} . E_{i}$ is calculated as follows.

Catchment areas with a one-mile radius centered around terminals $w_{1}, w_{2}, w_{3}$ and $w_{4}$ are created. The radius is configured to represent the maximum distance that most pedestrians are believed to be willing to walk to a transit stop. In previous studies, the radius of the catchment area has been ranging from a quarter-mile to 1.5 miles (Fayyaz et al., 2017; Flamm and Rivasplata, 2014; UTA, 2020; ACCESS Magazine, 2020) and results show that varying the size of the radius had very little influence on the ability to predict ridership based on the measures of surrounding characteristics (e.g., populations, jobs, etc.) Here we use a one-mile radius to compute the environmental equity around each transit station as a median value derived from previous studies. A one-mile radius roughly corresponds to the distance someone can walk in 20 minutes at three miles per hour to get to a transit station. We adopt it here to represent the geographic area where most disadvantaged populations will be exposed to air pollutants as they walk to or from a transit station. Thus, the union of the four catchment areas centered at $w_{1}, w_{2}, w_{3}$ and $w_{4}$ forms the influence area of bus $i . E_{i}$ is calculated as low-income population $\times$ pollutant concentration within this area.

The arriving sequence at $\mathrm{m}$ is denoted as $\alpha_{m}$, and in the above example, $w_{1}$ is assumed to be a potential site for building an on-route charging station, $\alpha_{w_{1}}$ is $\{(i, s=1),(i, s=$ $5)\}$. This indicates that bus $i$ stops at terminal $w_{1}$ twice. The sequence of terminals is 1 and 5 , respectively. $\beta_{m t}$ is a subset of $\alpha_{m}$, which represents the set of terminal sequences at time $t$. Buses that arrive around the same $\mathrm{t}$ (a time buffer centered at time point $t$ with the length of 10 minutes, which is $[t-5, t+5])$ are considered to conflict with each other, meaning they require simultaneous charging. $R$ is the range that a 
certain type of BEB can drive without charging. $T D_{i}$ is the accumulative mileage for bus $i$. $l_{i, s-1, s}$ is the actual driving distance from terminal $s-1$ to terminal $s$.

Binary decision variable $X_{i s}$ indicates whether bus $i$ is charged at terminal $s . D_{i s}$ is the total distance bus $i$ has traveled without being charged at terminal $\mathrm{s}$. If bus $i$ is charged at terminal $m_{0}$, then $X_{i m_{0}}$ is equal to 1 and $D_{i m_{0}}$ is set to zero. Binary decision variable $Z_{i}$ indicates whether bus $i$ is replaced with a BEB. Integer decision variables $Y_{m}^{O}$ and $Y_{n}^{I}$ represent the number of on-route charging stations built at $m$ and the number of indepot charging stations build at $n$, respectively.

Considering the unique spatiotemporal characteristic of BEBs and the goal to explore the trade-off between cost and environmental equity, formulation of the problem is as follows:

Bi-objective Battery Electric Bus Deployment Problem (BOBEBD)

Objective:

$$
\begin{aligned}
& \max \sum_{i} E_{i} Z_{i} \\
& \min \left(\sum_{i} C^{B} Z_{i}+\sum_{m} C_{m}^{O} Y_{m}^{O}+\sum_{m} C_{n}^{I} Y_{n}^{I}\right)
\end{aligned}
$$

\section{Subject to}

$$
\begin{aligned}
& D_{i, s-1}+l_{i, s-1, s} \leq R+\left(1-Z_{i}\right) T D_{i} \\
& D_{i, 1}=0, \forall i \\
& D_{i, s} \leq D_{i, s-1}+l_{i, s-1, s}, \forall i, s \geq 2 \\
& D_{i, s} \geq D_{i, s-1}+l_{i, s-1, s}-T D_{i} X_{i s}, \forall i, s \geq 2 \\
& D_{i, s} \leq\left(1-X_{i s}\right) T D_{i}, \forall i, s \geq 1 \\
& X_{i s} \leq Y_{m}^{O}, \forall m,(i, s) \in \alpha_{m} \\
& X_{i s} \leq Z_{i}, \forall i, s \\
& \sum_{(i, s) \in \beta_{m t}} X_{i s} \leq p^{o} Y_{m}^{O} \forall m, t \\
& \sum_{i \in \gamma_{n}} Z_{i} \leq p^{I} Y_{n}^{I} \forall n \\
& X_{i s}=0 \text { or } 1, \forall i, s
\end{aligned}
$$


$Z_{i}=0$ or $1, \forall i$

$Y_{m}^{O}$ and $Y_{n}^{I}$ are positive integers

$D_{i s} \geq 0, \forall i, s$

Objective (1) is to maximize environmental equity and (2) is to minimize the total costs of purchasing BEB and building charging stations. Constraints (3) guarantee that the mileage of a $B E B$ does not reach the maximum driving range before charging.

Constraints (4) ensure that the accumulating mileage is set to zero at the beginning of the day. Constraints (5) and (6) combined determine that if no charging takes place at terminal $s$, then the accumulative driving distance of bus $i$ at terminal $s$ is equal to the accumulative driving distance at terminal $s-1$ plus route distance between terminal $s-$ 1 and $s$. Constraints (7) set $X_{i s}$ to zero if bus $i$ gets charged at terminal $s$. Constraints (8) assure that a BEB can only be charged at a terminal if there are on-route charging stations built at that terminal. Constraints (9) relieve the driving limit on diesel or CNG buses and guarantee that only BEBs can be charged. Constraints (10) make sure that there are enough on-route charging slots for simultaneous charging while constraints (11) satisfy the need for overnight in-depot charging. Constraints (12) describe the nature of the decision variables.

To sum up, these constraints function jointly to ensure eligible buses will be replaced with BEBs to fulfill the existing designated routes and schedule.

BOBEBD is nontrivial. No single solution exists that simultaneously optimizes both objectives. Naturally, increasing the budget is very likely to lead to more BEB deployment, thus improving environmental equity. To seek solutions that are of practical value, constraint method (Cohen, 1978) is applied here. Objective (2) is therefore treated as a new constraint:

$$
\min \left(\sum_{i} C^{B} Z_{i}+\sum_{m} C_{m}^{O} Y_{m}^{O}+\sum_{m} C_{n}^{I} Y_{n}^{I}\right) \leq C_{x}
$$

$C_{x}$ is a variable that could be manually chosen to represent the actual budget imposed onto the entire system. With specific values of $C_{x}$, BOBEBD becomes a single objective mixed integer programming (MIP) problem, which can be solved using solvers like Gurobi, Cplex, GLPK, etc. We can vary $C_{x}$ to quantify how environmental equity would change accordingly and also observe different deployment plans, including which buses to replace, how many, and where charging stations are to be built.

\subsection{CASE STUDY IN UTAH}

This project is motivated by the need of the UTA to assess the feasibility of multiple BEB deployment plans in the next several years. The BOBEBD explores the possibility of pursuing a lower cost of replacing the system in phases while maximizing the 
environmental equity outcomes. UTA is responsible for providing public transportation services throughout the Wasatch Front of Utah in the United States, which includes the metropolitan areas of Ogden, Park City, Provo, Salt Lake City, and Tooele. UTA continues to expand its network since it was founded on March 3, 1970. The agency now has a coverage area servicing 2.2 million people, which accounts for almost $79 \%$ of the total population in the state. In 2016, UTA runs 467 diesel or CNG buses serving 121 routes on a typical weekday. In this study, traffic network data in 2016 is used. Fig. 1 shows the study area of this research, including bus routes and potential locations for both on-route and in-depot charging stations. Many buses operate trips on two or more routes in one day (interlining) in order to maximize the efficiency of operations by offering a flexible solution of pairing the schedules for both drivers and buses.

The specific type of BEB under consideration in this study is New Flyer's XE40, five of which are currently serving Salt Lake City and the University of Utah campus. The parameters referenced here are collected from their operation data. Based on their current operation, the driving range of XE40 varies from 62 miles to 200 miles in winter and from 75 miles to 294 miles in summer depending on the intensity of battery usage. During wintertime, the battery is mainly consumed by the motor and electric heater. Using the operational records between January 1st, 2020, and January 15th, 2020, the electric heater could take up to $50 \%$ of battery consumption, which hindered the fullcharging driving range significantly. During summertime, the air conditioning could also take up a considerable amount of battery consumption, yet it is less than $50 \%$ even in the most extreme cases. Moreover, considering the elevation rise along several routes in Utah, a safe assumption of 62 miles is made in this study. Under this assumption, there are 114 buses among a total of 467 having a daily mileage less than 62, indicating no on-route charging is needed and 51 buses will run out of battery before charging due to the long distance between stops. The standard charging time for XE40 using on-route charging is $10-13$ minutes. No partial charging is assumed in this study. Thus, only terminals in which any bus dwells more than 10 minutes will be deemed as potential sites for building on-route charging stations. This results in 71 potential charging stations for the study region and four bus garages in the Wasatch Front are qualified as in-depot charging stations for overnight charging without space limitations (Figure 3.1). Among the remaining 302 buses operated by UTA on weekdays, 82 cannot be fully charged because they dwell less than 10 minutes at any terminals, which means they are not qualified as replacements given the current parameters. It leaves 220 buses in total that require in-depot charging and on-route charging. 


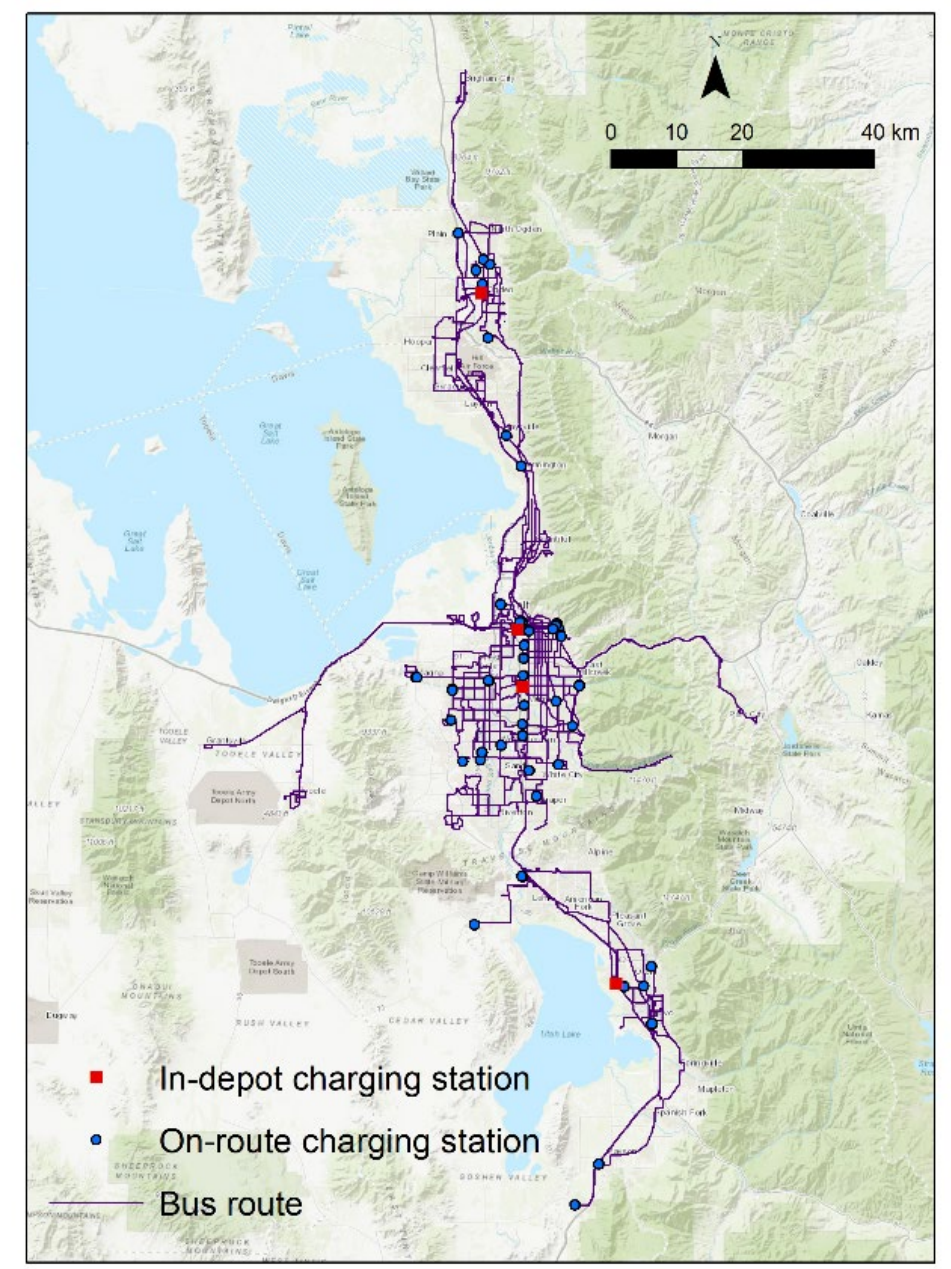

Figure 3.1: Study Area

To sum up, $334(220+114)$ buses are considered in the BOBEBD. According to UTA, an in-depot charging station can charge up to three buses simultaneously and an onroute charging station can only charge one bus at a time. The construction costs for indepot and on-route charging stations are $\$ 350,000$ and $\$ 1$ million, respectively. The cost of purchasing one XE40 is $\$ 790,000$.

\subsubsection{Air Pollution Data}

In order to model the environmental equity outcomes as a result of BEB deployment, air population data needs to be collected. We obtain such information from PurpleAir (2020). PurpleAir is an air quality monitoring network built on a new generation of laser particle counters to provide real-time measurement of PM1.0, PM2.5, and PM10. The PurpleAir sensors are mainly installed in Europe and North America, and there are over 400 public sensors distributed across Utah. Figure 3.2 shows a sample screenshot for PurpleAir Air Quality Index (AQI) reading on April 8th, 2020, in Utah. The data feed from PurpleAir includes both real-time and weekly averages of particulate matter (PM1.0, 
PM2.5, PM10) concentration every five minutes. In Utah, PurpleAir sensors are highly congregated in the urbanized areas, such as Salt Lake City.

Major pollutants generated out of vehicular traffic, such as NOx and CO, are not used for indicators as the emission caused by road traffic is not the focus in our research, yet the focus is to deploy BEBs in such a way that the low-income populations who are exposed to the worst air conditions can be given priority. To this end, the concentration of PM2.5 $\left(\mathrm{ug} / \mathrm{m}^{3}\right)$ is treated as the indicator of air pollution level in this study. The reason for using PM2.5 as a measure of air quality is because the Greater Salt Lake region is classified as a nonattainment area for PM2.5 by the Environmental Protection Agency for 11 years in a row since 2009 (U.S. Environmental Protection Agency, 2020). We retrieved PM2.5 concentration from all sensors in the state of Utah from October 1st to October 14th, 2019, and calculated the average for each site. The data was further processed to interpolate the pollutant level at the unit of Traffic Analysis Zone (TAZ).

This will be explained in detail in Section 3.2.3.

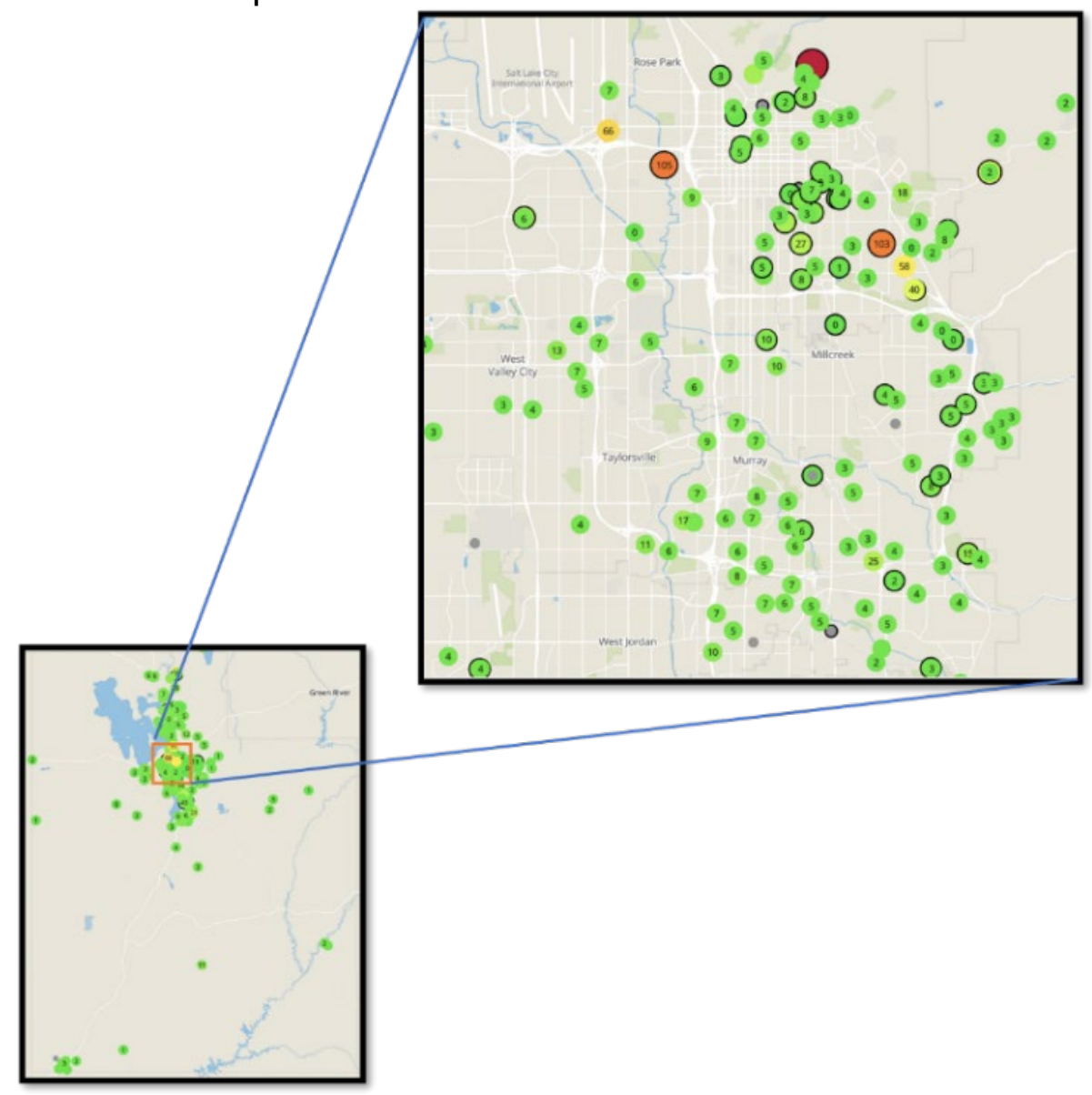

Figure 3.2: Sample Screenshot of PurpleAir Sensor Distribution in the State of Utah on $04 / 08 / 2020$

\subsubsection{Low-Income Population}

The low-income population is retrieved from metropolitan planning organizations (MPOs) in Utah for the year 2019. All households were first classified into four groups 
according to the 2010 Census income groupings. The income group 1 ranging from $\$ 0$ to $\$ 34,999$ is treated as the low-income group for Utah. The distribution of the lowincome population at TAZ level is shown in Fig. 3. Note that several issues might occur when using Census data. First of all, an even distribution of the population within TAZ is assumed. Without acquiring additional information, such as land use and points of interest, such an assumption might over/underestimate $E_{i}$. Furthermore, considering only the residents-based indicators (e.g. low-income residents) can potentially underestimate the number of served populations as well. This is because the number of people using transit services in certain areas might not be the same people or a reflection of the number of people residing in those areas. While the fundamental assumption that low-income populations are heavily dependent on public transit for mobility and fulfilling their daily activities holds, as verified by other studies such as Fayyaz et al. (2017), combining residents-based data with land use or other detailed human activities data could further enhance the accuracy of the estimation.

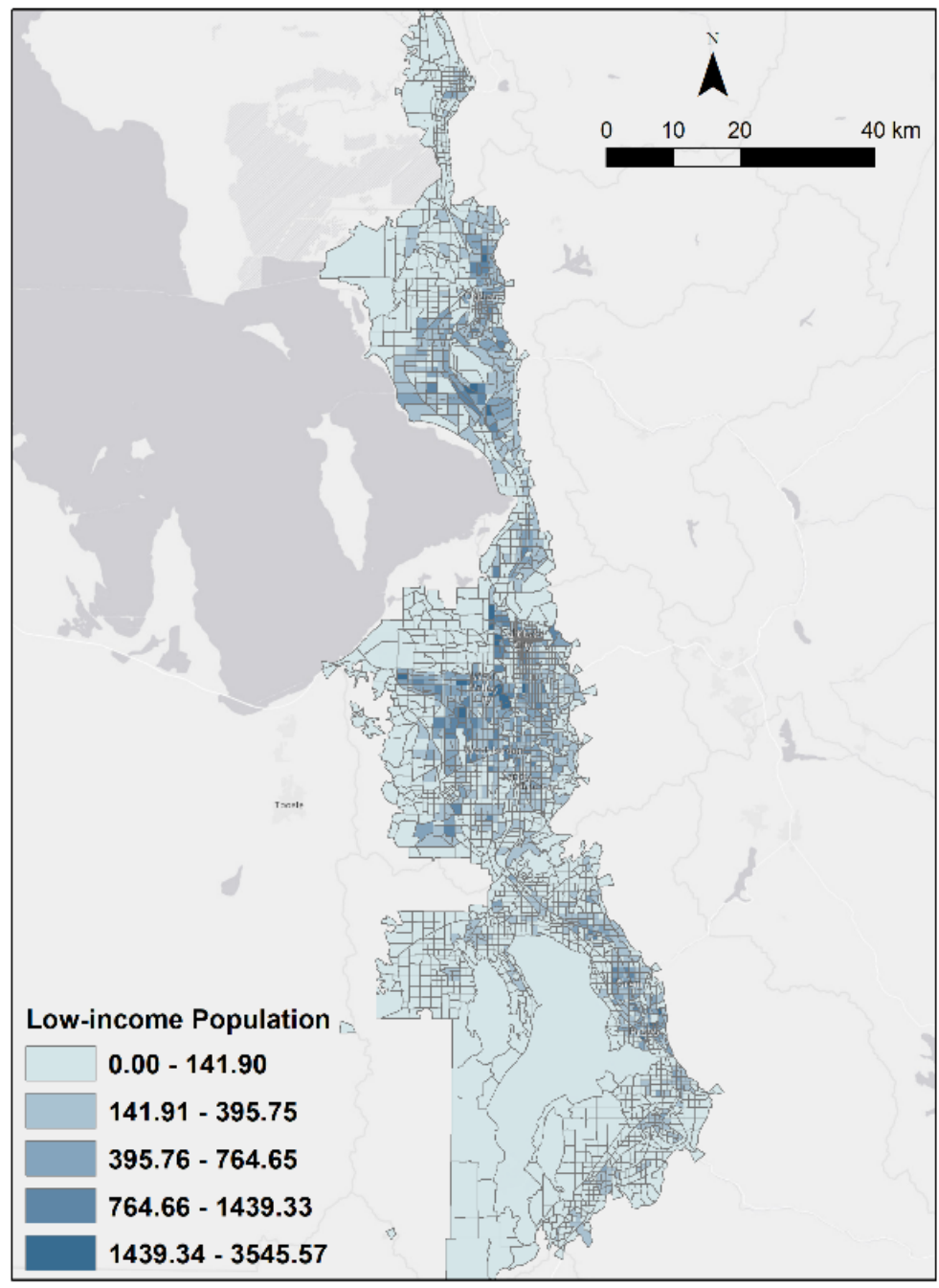

Figure 3.3: Distribution of Low-Income Populations 


\subsubsection{Calculation of $E_{i}$ for Each Bus}

Before solving BOBEBD, $E_{i}$, environmental equity reached by replacing bus $i$ is calculated. Note in Figure 3.2 that the PurpleAir monitoring sites are not evenly distributed across Utah; therefore, data processing is needed to interpolate the PM2.5 values across the entire geographical surface. Ordinal kriging (Cressie, 1990) is applied here to create a smooth surface of PM2.5 concentration. Kriging is a method of interpolation for which the interpolated values are modeled by a Gaussian process. In this research, the Gaussian semivariogram model is chosen and cell size for kriging will need to be specified. Since the low-income population is aggregated by TAZ, the cell size is adjusted such that each TAZ contains at least one centroid from the raster created via kriging. For UTA's network, the cell size is set as 600 feet. Figure 3.4 shows the resulted average PM2.5 concentration delineated by TAZ. Comparing Figure 3.3 and Figure 3.4, it is noted that most of the low-income population resides in TAZs with higher PM2.5 concentration. For example, in central Salt Lake City where PM2.5 concentration is the highest, there is a cluster of TAZs with a larger low-income population which accounts for more than $50 \%$ of the total low-income populations in the studied region. Also, the region to the east of the Great Salt Lake shows similar patterns where low-income populations reside in areas with a higher concentration of PM2.5.

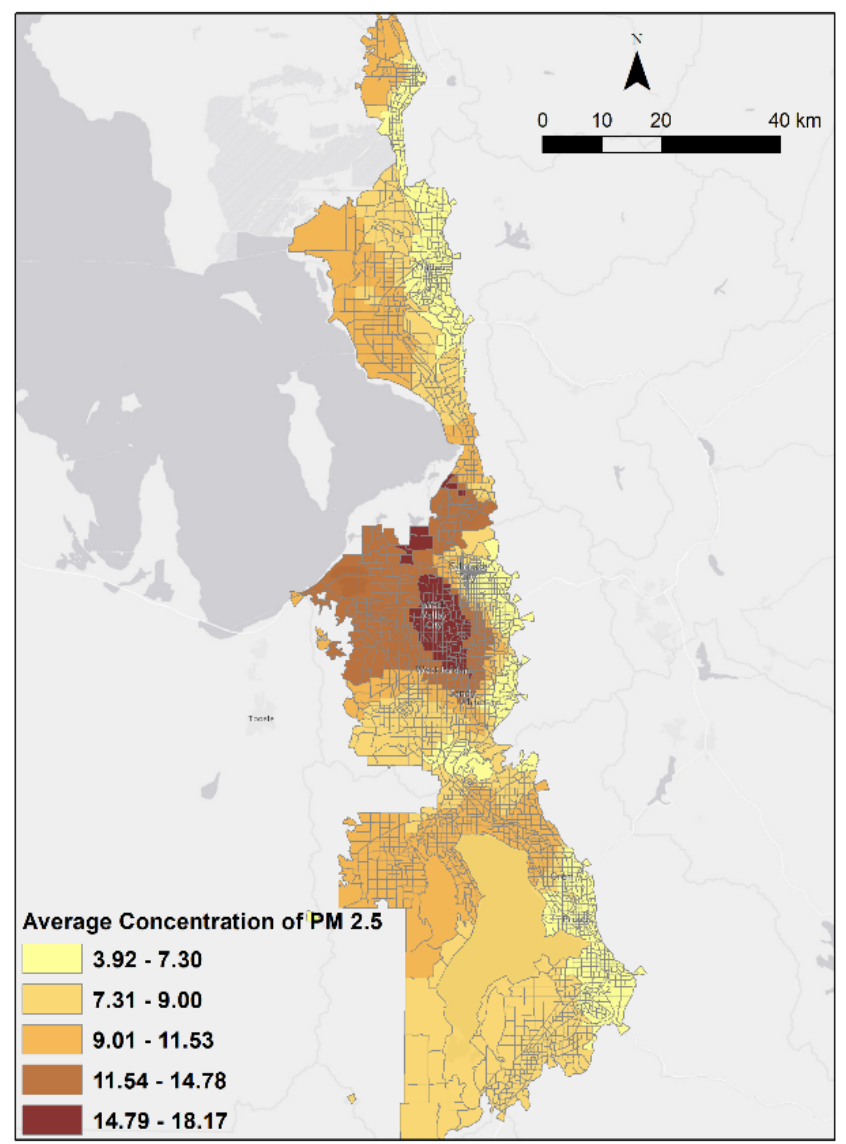

Figure 3.4: PM2.5 Concentration Delineated by TAZ for Utah 
Correspondingly, the average concentration of PM2.5 can be calculated for $T A Z_{j}$, where $j$ is the index of TAZs. The concentration of PM2.5 in $T A Z_{j}$ is referred to as $P M_{T A Z_{j}}$ and the population in $T A Z_{j}$ is referred to as $P O P_{T A Z_{j}}$. Then, the low-income population weighted by PM2.5 concentration is calculated as:

$$
W P O P_{T A Z_{j}}=P M_{T A Z_{j}} \times P O P_{T A Z_{j}}
$$

The calculation of $E_{i}$ is demonstrated using Route No. 205 in Utah. Figure 3.5 shows the stops, route, and influence area of Route 205 as well as the TAZs. It's assumed that bus $i$ operates on Route No. 205 during trip $h$, where $h$ is the index of trips. $W$ stops are visited sequentially by bus $i$ during trip $h$, which is denoted as grey points in Figure 3.5. A catchment area with a one-mile radius was created at each one of the $W$ stops. The union of the $W$ catchment areas is then obtained, which is referred to as $U_{i}^{h}$. $U_{i}^{h}$ is the blue region in Figure 3.5 representing the influence area of Route No. 205. Then $U_{i}^{h}$ is further intersected with all TAZs. The boundaries of TAZs and $U_{i}^{h}$ generates the unit for calculating the weighted population. The proportion of $T A Z_{j}$ contained in $U_{i}^{h}$ can be calculated as a result, which is referred to as $\operatorname{Prop}_{i}^{h}\left(T A Z_{j}\right)$. The weighted population contributed by $T A Z_{j}$ is calculated as such:

$$
\operatorname{Prop}_{i}^{h}\left(T A Z_{j}\right) \times W P O P_{T_{A Z}}
$$

Then for the specific trip $h$ operating on Route 205, traversing multiple TAZs (entire set $J)$, this indicator is calculated as:

$$
\sum_{j \in J} \operatorname{Prop}_{i}^{h}\left(T A Z_{j}\right) \times W P O P_{T A Z_{i}}
$$

Since bus $i$ operates on Route No. 205 multiple times during the day, the entire set of trips made by bus $i$ on a weekday is denoted as $H$. Then,

$$
E_{i}=\sum_{h \in H} \sum_{j \in J} W P O P_{T A Z_{i}} \times \operatorname{Prop}_{i}^{h}\left(T A Z_{j}\right)
$$




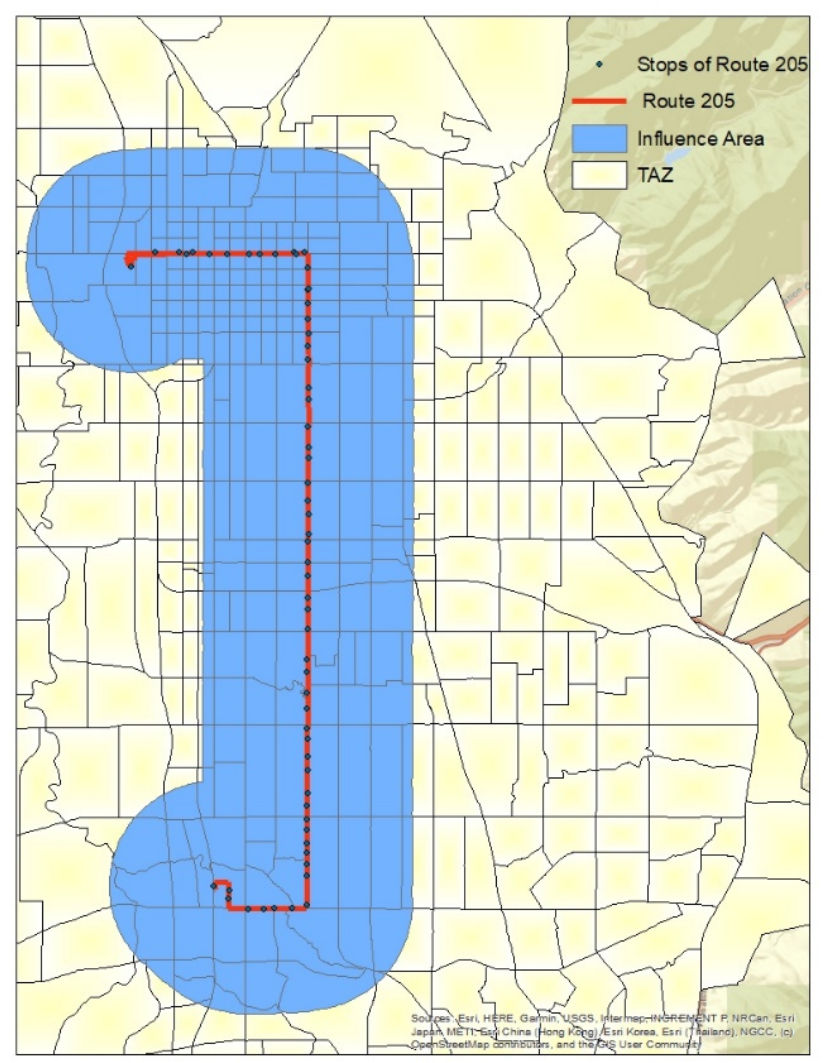

Figure 3.5: Illustration for $E_{i}$ Computation of Bus $i$

The distribution of $E_{i}$ associated with all the buses is shown in Fig. 6. $E_{i}$ associated with the majority of buses is below $25,000 \mathrm{ug} / \mathrm{m}^{3}$, which accounts for more than $90 \%$ of the total fleet.

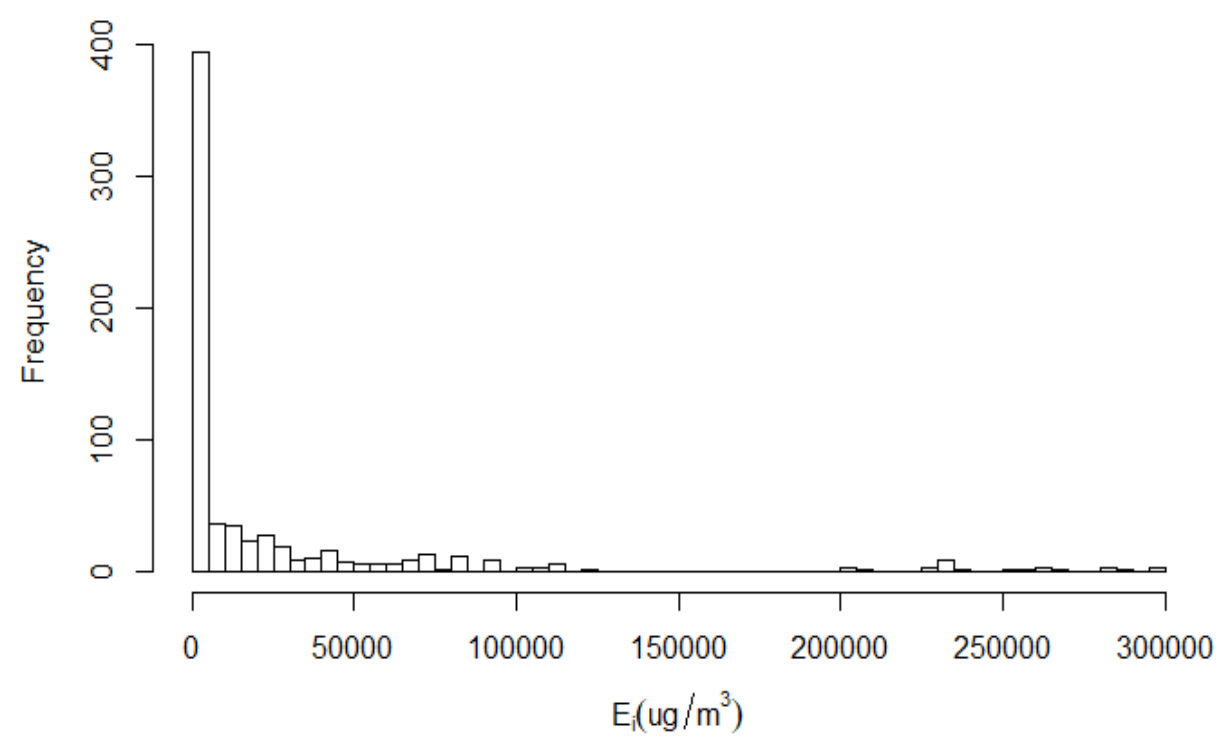

Figure 3.6: Distribution of $E_{i}$ 


\subsection{CASE STUDY IN OREGON}

The methods presented in this project are also applied into planning the BEB conversion process of the diesel buses operated by TriMet - the Tri-County Metropolitan Transportation District of Oregon, in the Portland metropolitan region. TriMet provides bus, light rail, and commuter rail spanning Multnomah, Washington, and Clackamas counties, operating in a region of about 533 square miles. TriMet's annual proposed budget for 2021 totals $\$ 1.64$ billion (TriMet, Proposed Fiscal Year Budget, 2020). An estimated 316,700 trips are taken on the TriMet transit system each day. On September 26, 2018, TriMet's Board of Directors approved a plan to fully transition to an all-electric bus fleet by 2040 (Metro, 2019). TriMet purchased five BEBs using $\$ 3,405,750$ awarded from the Federal Transit Administration's (FTA) Low or No Emission Vehicle Program in 2016, and began its first BEB testing in 2019 along Line 62-Murray Blvd. TriMet was awarded another $\$ 2,088,579$ from the FTA in 2019 , and plans to use it combined with funding from Oregon House Bill 2017 to purchase 15 more BEBs. TriMet's current plan is to purchase 70 BEBs over the next four years (TriMet, TriMet Electric Bus Plan, 2019).

In 2018 , TriMet reported that $97 \%$ of its buses were powered by diesel fuel; $65 \%$ of that fleet was equipped with selective catalytic reduction (Trimet, 2018). The BOBEBD is employed to determine the optimal deployment strategies for the BEB system within certain budget parameters. The bus fleet operation schedule, bus routes, and bus terminals are supplied by TriMet and based on the Spring 2020 operation schedule.

The specific BEBs purchased by TriMet are New Flyer's 40-foot Xcelsior Charge XE40 model that can reach a range of up to 130 miles during summer months and around 85 miles in the winter at full-charge capacity. The on-route, ABB HVC $450 \mathrm{~kW}$ Pantograph Charger takes between three to six minutes to recharge each BEB; therefore, BEBs can only be charged at a terminal if it dwells there for more than six minutes. The model assumes there will be no partial charging. Given these constraints, only seven of the existing buses are unable to be charged before running out of battery if they are replaced with the XE40 BEBs. This leaves 826 buses possible for BEB replacement. The cost of purchasing an XE40 is $\$ 915,856$. Bus terminals are considered as potential locations for on-route charging stations if one or more buses stop there for more than six minutes. This results in 194 potential sites for on-route charging stations. All three bus garages are considered as potential sites for in-depot charging stations. Given the requirement of a six-minute charging time, any buses that arrive at the same terminal within the same six-minute charging window are considered to conflict since only one of them will be able to charge at the terminal. The on-route charging stations used by TriMet can only charge one bus at a time and each in-depot charging station can provide a full charge to three BEB overnight. The assumed cost to build an on-route charging station at a bus terminal is $\$ 584,000$, and the cost to install an in-depot charging station for each bus is $\$ 69,000$. 


\subsection{RESULTS AND ANALYSIS}

\subsection{SALT LAKE CITY, UTAH}

\subsubsection{Trade-off between Costs and Environmental Equity}

The BOBEBD is solved by introducing constraints (13) for varying $C_{x}$. The intention is to identify the trade-off between environmental equity reached by replacing BEBs and the cost. $C_{x}$ can be well interpreted as budget constrained by funding availability. Different deployment plans can be presented by varying $C_{x}$. Each plan would produce the set of locations for charging stations and replaced buses, given a fixed $C_{x}$ and the maximum environmental equity it could yield. Figure 4.1 shows the trade-off curve between budget $C_{x}$ and environmental equity each unique plan could produce. There is a clear positive correlation between budget and environmental equity. As the budget rises, the number of buses applicable for replacement goes up as well as the number of on-route and indepot charging stations.

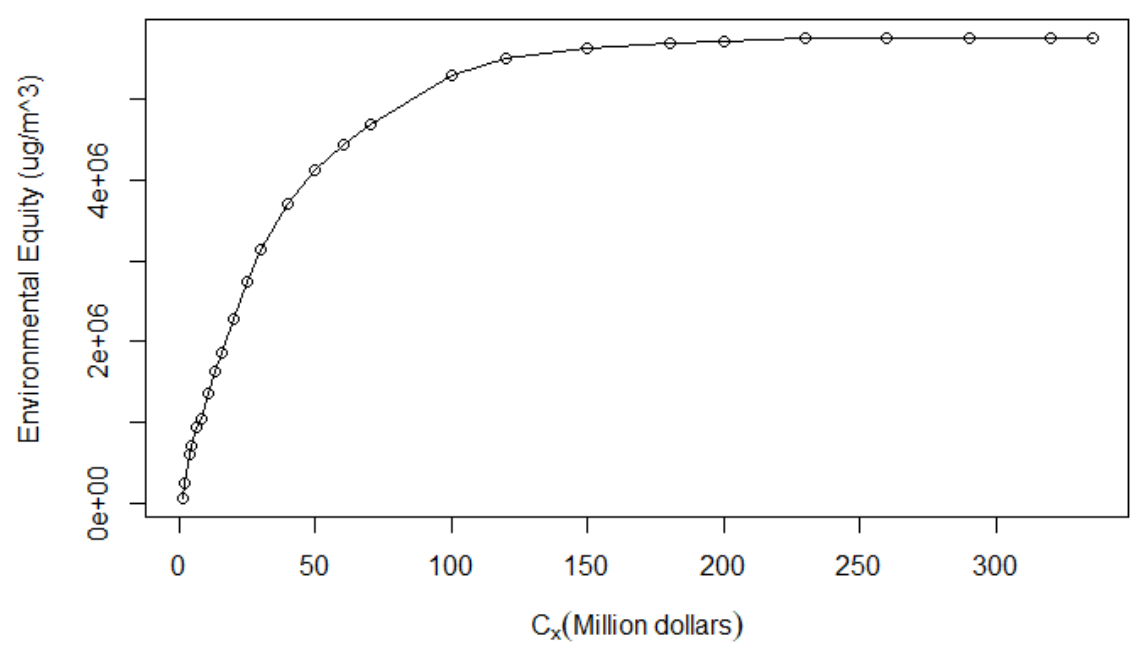

Figure 4.1: Trade-off Curve between Cost and Environmental Equity

There are potentially $334(220+114)$ buses feasible for replacement and 114 of them do not require on-route charging stations. The maximum environmental equity that could be achieved would correspond to the scenario where all buses are replaced with BEBs. Such a scenario requires 46 on-route charging stations and 112 in-depot charging stations to be installed. The total cost for BEBs and charging stations is $\$ 335.366$ million whose environmental equity reached is $5.76 \times 10^{6} \mathrm{ug} / \mathrm{m}^{3}$.

\subsubsection{Examples of Deployment Plans}

Figure 4.2 shows the deployment plan while the budget is set as $\$ 25$ million, which is approximately $13 \%$ of the total cost for replacing all buses. The environmental equity achieved is $2.75 \times 10^{6} \mathrm{ug} / \mathrm{m}^{3}$, which is around $47.7 \%$ of the scenario when all buses are 
replaced with BEBs. While $C_{x}=\$ 25$ million, 26 buses are replaced, two on-route charging stations and nine in-depot charging stations are built. These 26 buses all require on-route charging and serve 11 routes whose distances range from 6.88 miles to 18.90 miles with an average of 11.48 miles. The two on-route charging stations are sited at West Valley Central Station (3650 S 2880 W) and Millcreek (Wasatch Blvd at $3900 \mathrm{~S}$ ). The daily mileage of the buses ranges from 161.89 miles to 263.33 miles, with an average of 202.98 miles. It is worth mentioning that $E_{i}$ associated with the 26 buses are ranked in the top $13 \%$ among all buses in $E_{i}$, ranging from the 13th to the 61 st among the 467 buses. The reason why buses with the highest $E_{i}$ are not selected is that those buses tend to operate on longer routes which require one or more on-route charging stations, and the difference in $E_{i}$ among the top $20 \%$ of buses is not significant.

If $C_{x}=\$ 60$ million, 63 buses will be replaced with BEBs while five on-route charging stations and 21 in-depot charging stations will be built. Figure 4.2 demonstrates the actual deployment plan. It brings $4.44 \times 10^{6} \mathrm{ug} / \mathrm{m}^{3}$ environmental equity outcome, which is $77.1 \%$ of the total environmental equity that the system can possibly achieve. Two out of five on-route charging stations are built at Millcreek (Wasatch BLVD at 3900S), while the other three are located at three different terminals in West Valley Central Station (3650 S 2880 W), North Temple Station (490 W 240 N), and Salt Lake Central Station ( $300 \mathrm{~S} 600 \mathrm{~W}$ ). The 63 buses replaced serve 20 routes whose distances range from 5.45 miles to 18.90 miles, with an average of 10.63 miles. The daily mileage of the 63 buses ranges from 62.78 miles to 263.33 miles, with an average of 176.20 miles. Furthermore, if the budget is raised to $\$ 120$ million, 122 buses are to be replaced with 14 on-route charging stations and 41 in-depot charging stations built, which brings $5.51 \times 10^{6} \mathrm{ug} / \mathrm{m}^{3}$ environmental equity and accounts for $95.7 \%$ of the total. As shown in Figure 4.4, the 14 on-route charging stations are located across the region in Millcreek, West Valley, Salt Lake, South Salt Lake, Sandy, South Ogden, Orem, and Murray. The 122 buses replaced serve 32 routes whose distances range from 5.45 miles to 23.15 miles, with an average of 11.53 miles. The daily mileage of the 122 buses ranges from 62.78 miles to 263.33 miles, with an average of 170.52 miles. It is noticeable that the increase in environmental equity brought by replacing additional BEBs drastically declines because buses that could reach the most environmental equity are already included in the first 63 buses. If we continue to raise the budget to $\$ 200$ million, $99.3 \%$ of the total environmental equity will be reached, with 203 buses replaced and 24 onroute charging stations and 68 in-depot charging stations built. 


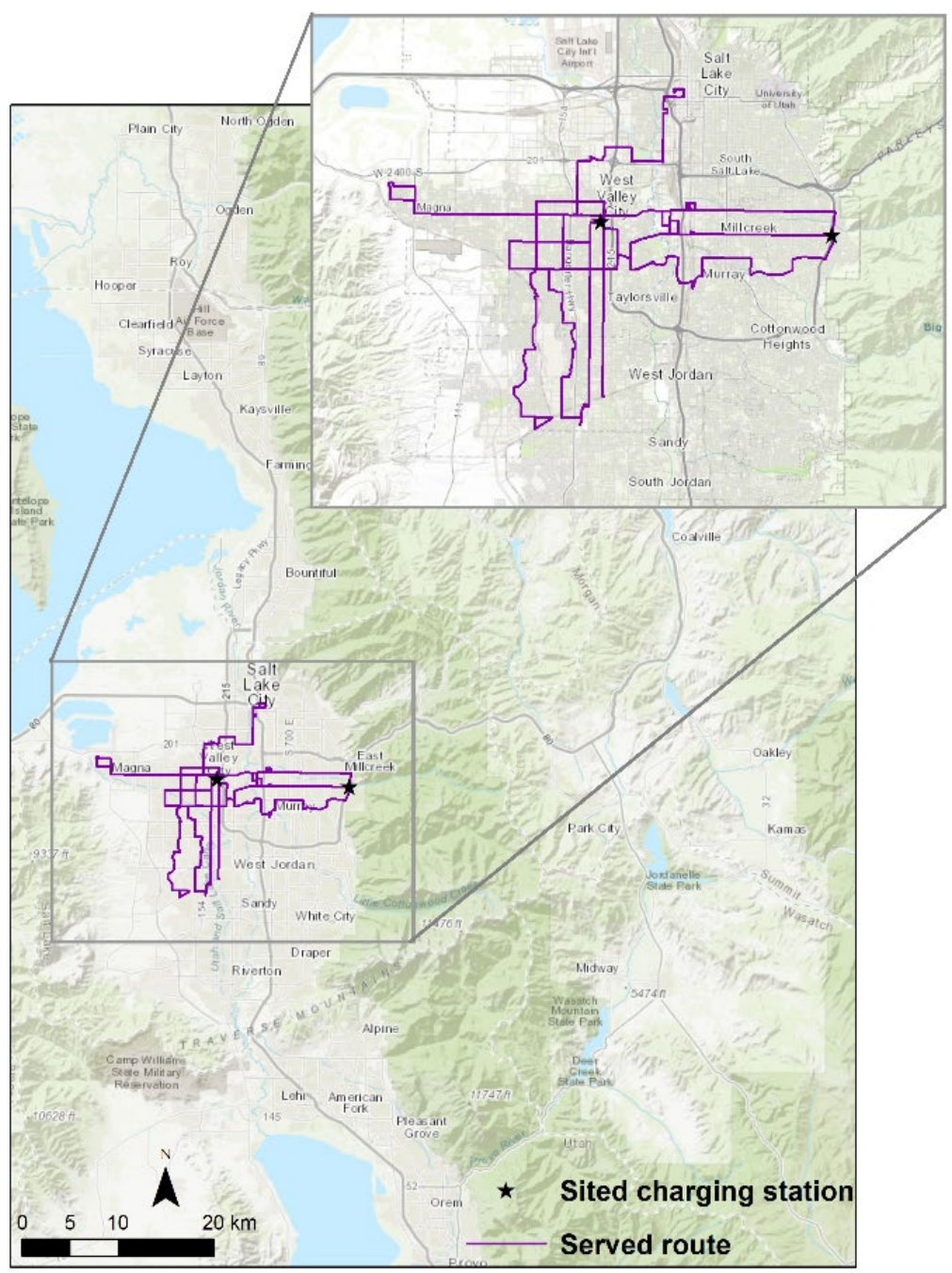

Figure 4.3: BEB Deployment Plan when Budget is set at $\$ 25$ million 


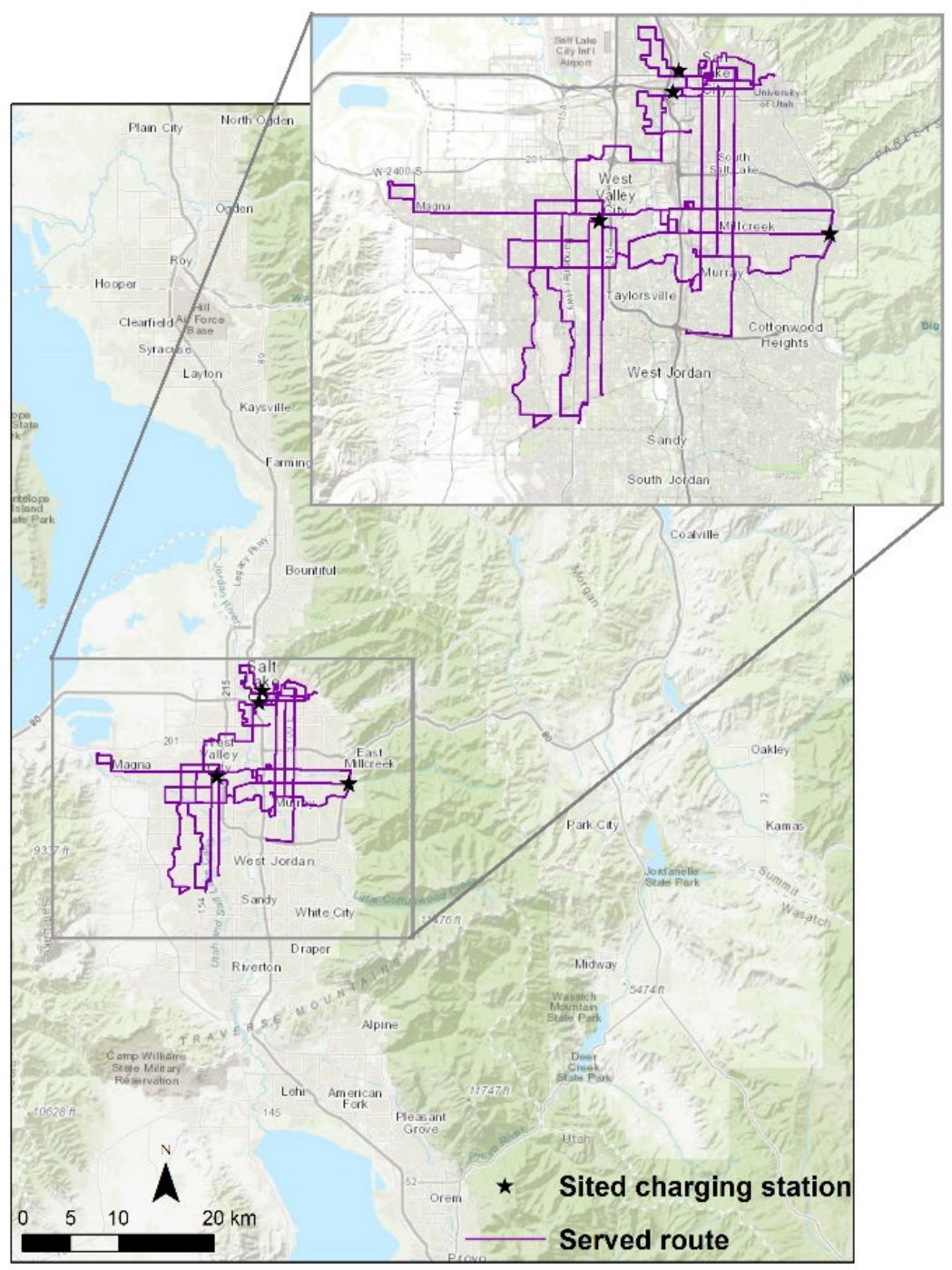

Figure 4.4: BEB Deployment Plan when Budget is set at $\$ 60$ million 


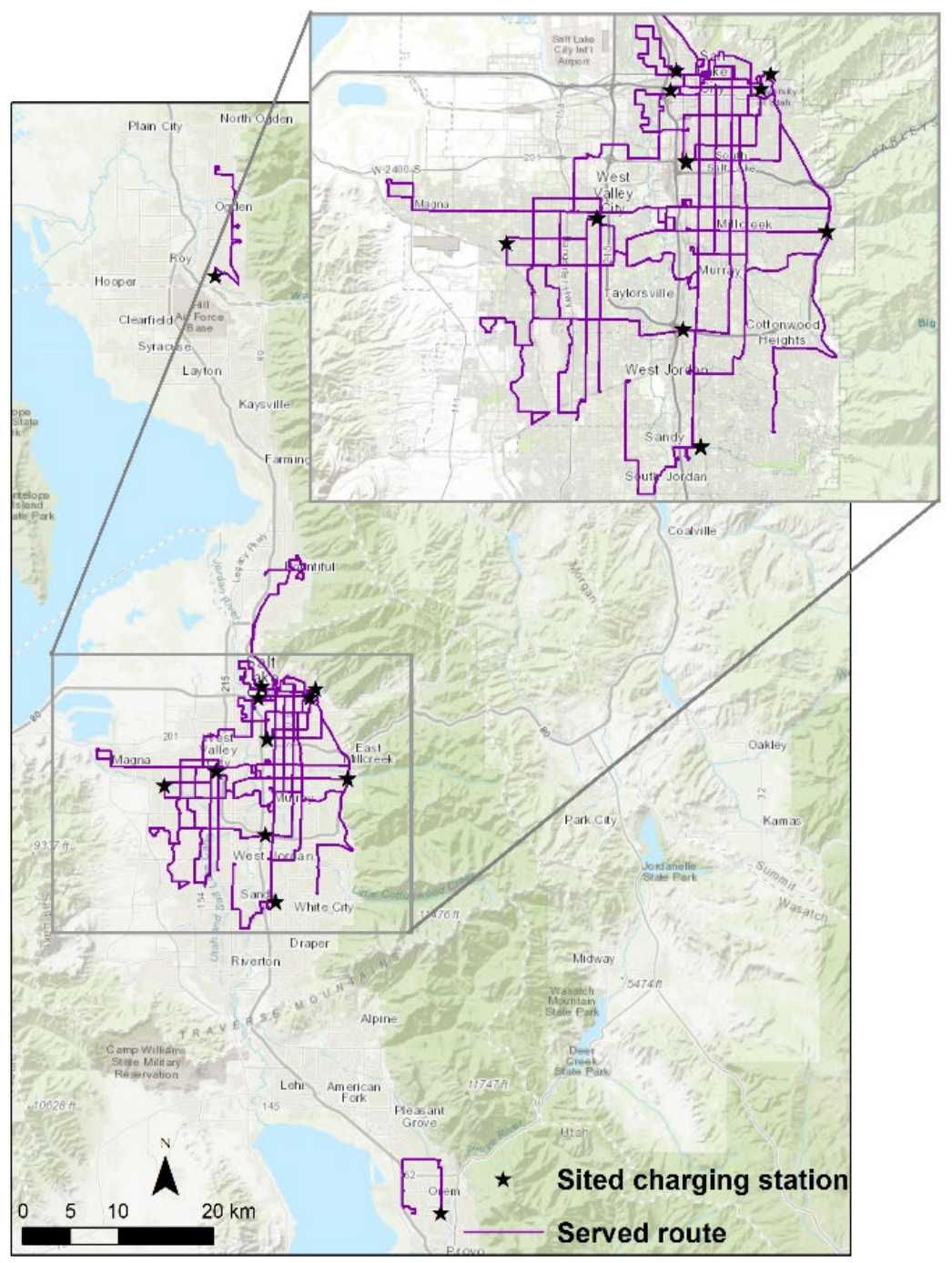

Figure 4.5: BEB Deployment Plan when Budget is set at $\$ 120$ million

\subsection{PORTLAND, OREGON}

\subsubsection{Examples of Deployment Plans}

Results from the multi-objective optimization model, BOBEBD, indicate a potential of 826 diesel buses that are feasible for replacement with BEBs in Portland -422 do not require on-route charging stations. The maximum possible environmental equity score, $E_{i}$, would be achieved if all feasible buses are replaced with BEBs. To replace all feasible buses, 87 in-route charging stations and 276 in-depot chargers (one for every three buses) are needed to support the new BEB fleet. The total cost for replacing all 826 buses, along with all supportive charging infrastructure, is $\$ 826,349,056$ and would achieve $2.45 \times 10^{7} E_{i}$ as the maximum environmental equity score. 
If TriMet follows through with its current plan to purchase 70 BEBs by 2022 (see in Table 4.1), the following bus, route, and in-route charging configurations would maximize the environmental benefits to low-income households in Portland in accordance with TriMet's stated equity goals.

Table 4.1: TriMet's Plan to Purchase 70 BEBs by 2022

\begin{tabular}{|l|c|l|}
\hline Year & BEB Replacements & Funding Source \\
\hline 2016 & 5 & FTA Low-No Grant \\
\hline 2019 & 15 & FTA Low-No Grant + HB2017 \\
\hline 2020 & 10 & HB2017 \\
\hline 2021 & 20 & HB2017 \\
\hline 2022 & 20 & HB2017 \\
\hline
\end{tabular}

To maximize environmental equity while ensuring the optimal scenario for replacing five TriMet buses with BEBs (see Figure 4.5), the BOBEBD model returns an optimal solution that would require two charging stations: one at the Clackamas Town Center MAX Station and one at SE Foster \& 94th (I-205 overpass). The five bus blocks that are selected for replacement are $7103,7106,7242,7244,1407$ serving the following routes: 14 (Hawthorne), 71 (60 ${ }^{\text {th }}$ Ave), 72 (Killingsworth/82 ${ }^{\text {nd }}$ ), 79 (Clackamas/Oregon City), and 155 (Sunnyside). The cost for this BEB replacement scenario is $\$ 5,885,280$ and would account for near $2 \%$ of the maximum environmental equity impact score.

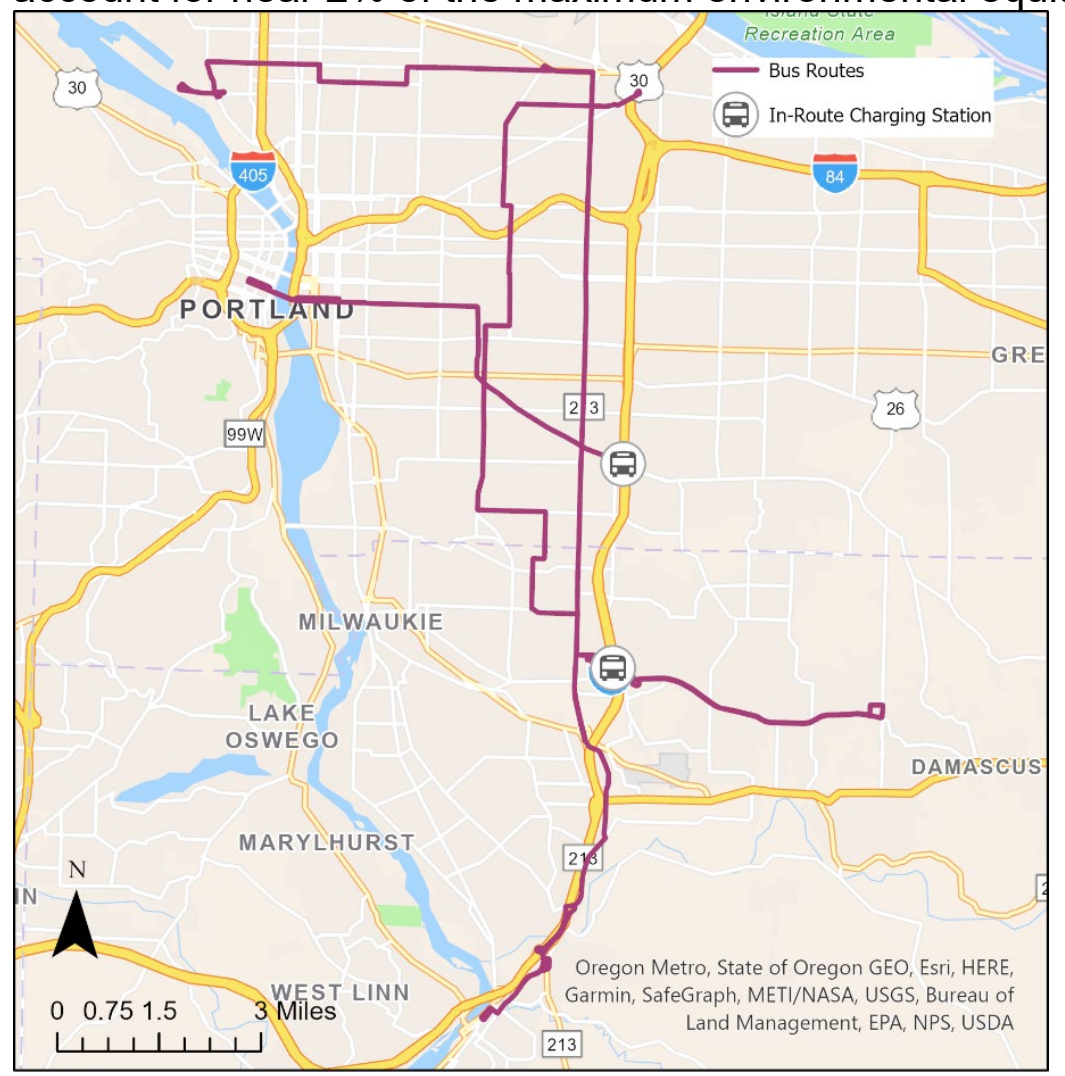

Figure 4.6: Maximum Environmental Equity with Five BEB Replacements 
As TriMet makes the transition to $20 \mathrm{BEB}$ replacements (see Figure 4.6) with funding sources from 2019, as their plan states, the BOBEBD model returns a solution that keeps four of the replacements from the initial five BEB configuration but does not include block number 1407 which runs along route 14 (Hawthorne). Instead, the model adds four blocks to the 71 and 72 route plan (blocks 7101, 7104, 7240, and 7246) and then focuses on routes 2 (Division) and 9 (Powell Blvd) which run east towards Gresham (bus blocks 201, 203, 204, 235, 236, 238, 243, 902, 934, 935, 937, and 943). This BEB replacement scenario includes two charging stations: one at the NE $10^{\text {th }}$ \& Hood stop in Gresham and one at the Clackamas Town Center MAX Station. The cost of this BEB replacement scenario is $\$ 19,968,120$ and it accounts for about $6.7 \%$ of the environmental equity score possible if all feasible buses are replaced by BEBs. If 30 buses are replaced with BEBs (see Figure 4.7), as is TriMet's goal for 2020, the trend continues that replacements occur on bus routes serving East Portland. This replacement configuration would see the addition of BEB buses serving routes 15 (Belmont/NW 23rd), 19 (Woodstock), 25 (Glisan/Rockwood), 22 (Parkrose), 24 (Fremont/NW 18 ${ }^{\text {th }}$ ), and one additional charging station at the Gateway Transit Center (three charging stations total). Additional bus blocks included in this configuration are $2235,2467,2468,1512,1901,1903,1904,1906$, and 1908. The cost of this scenario is $\$ 29,917,680$ and it accounts for $9.8 \%$ of the total possible environmental equity score.

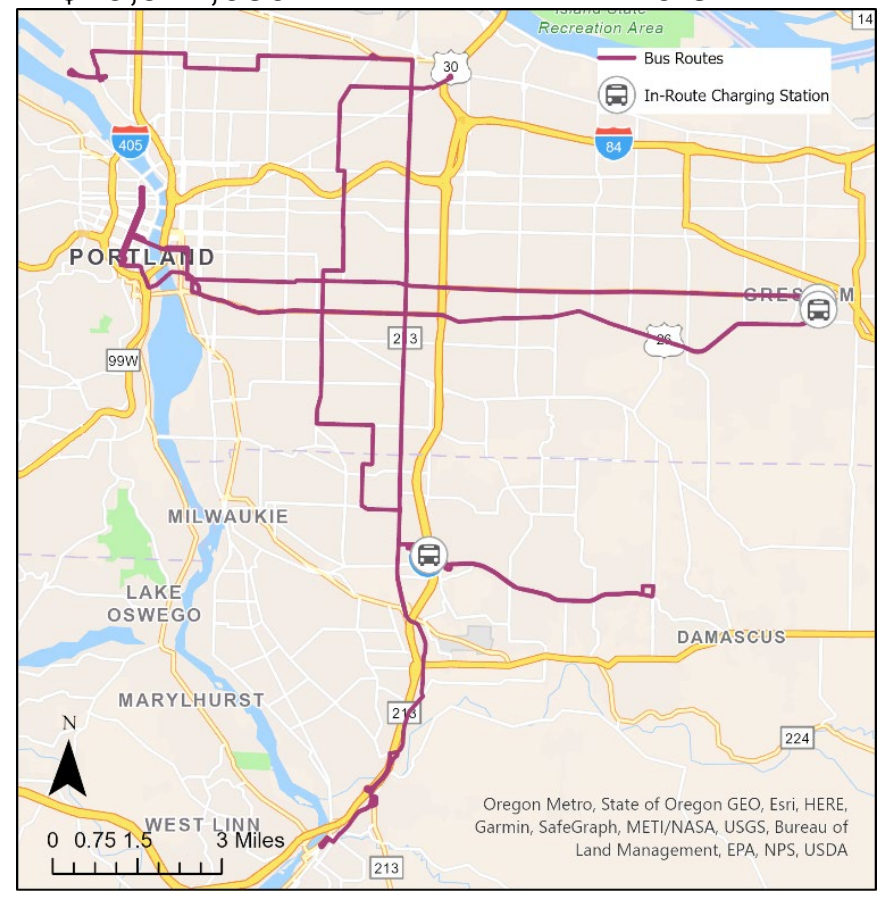

Figure 4.7: 20 BEB Replacements 


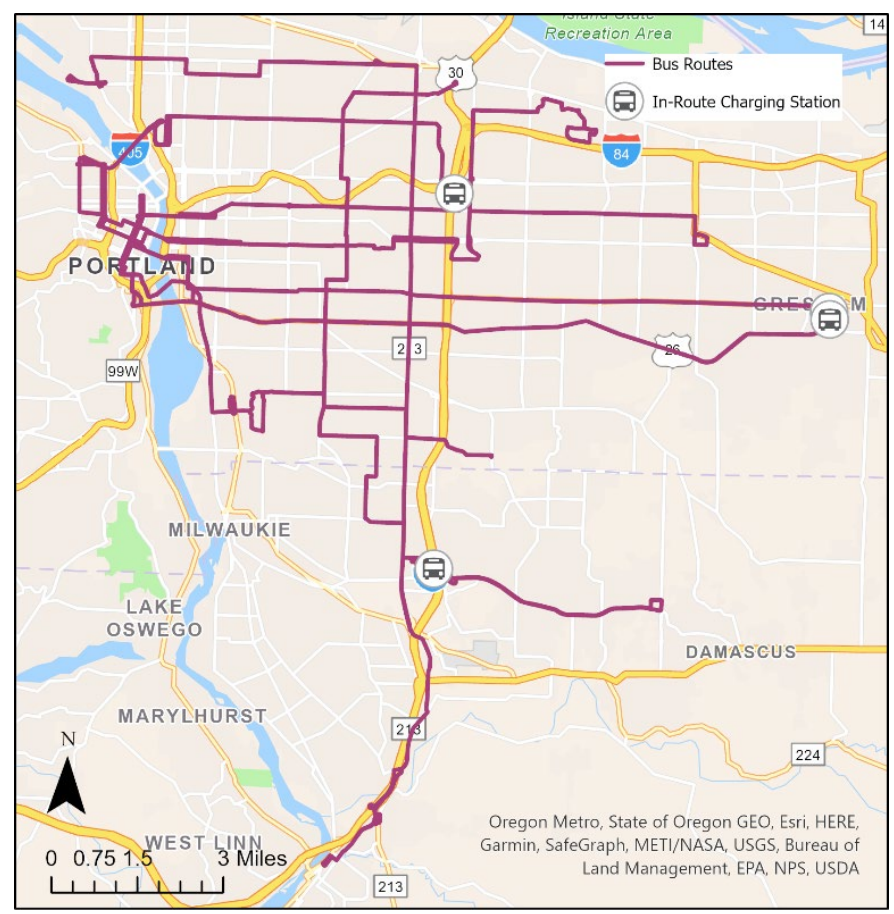

Figure 4.8: 30 BEB Replacements

With 50 BEB replacements (see Figure 4.8), we see additional bus blocks that serve the eastside as well as some in the west towards Beaverton like route 76 (Hall/Greenburg) and 54 (Beaverton-Hillsdale Hwy). Two lines, the 20 (Burnside) and 12 (Barbur/Sandy) run long routes across the city that span deep into the eastern and western suburbs. Two additional charging stations are required for this BEB arrangement: one at the Beaverton Transit Center and one at NW $6^{\text {th }}$ and Flanders (five charging stations total). The cost of this configuration is $\$ 49,885,800$, accounting for $15.9 \%$ total Ei. If 70 buses are replaced with BEB (Figure 4.9), according to TriMet's plan, bus replacements continue to prioritize the eastside. This configuration requires two additional charging stations: one at Parkrose/Sumner Transit Center and one at SE Holgate \& $134^{\text {th }}$ (seven total chargers). The cost of this scenario is $\$ 69,853,920.00$ and accounts for $21.7 \%$ total potential $E_{i}$. 


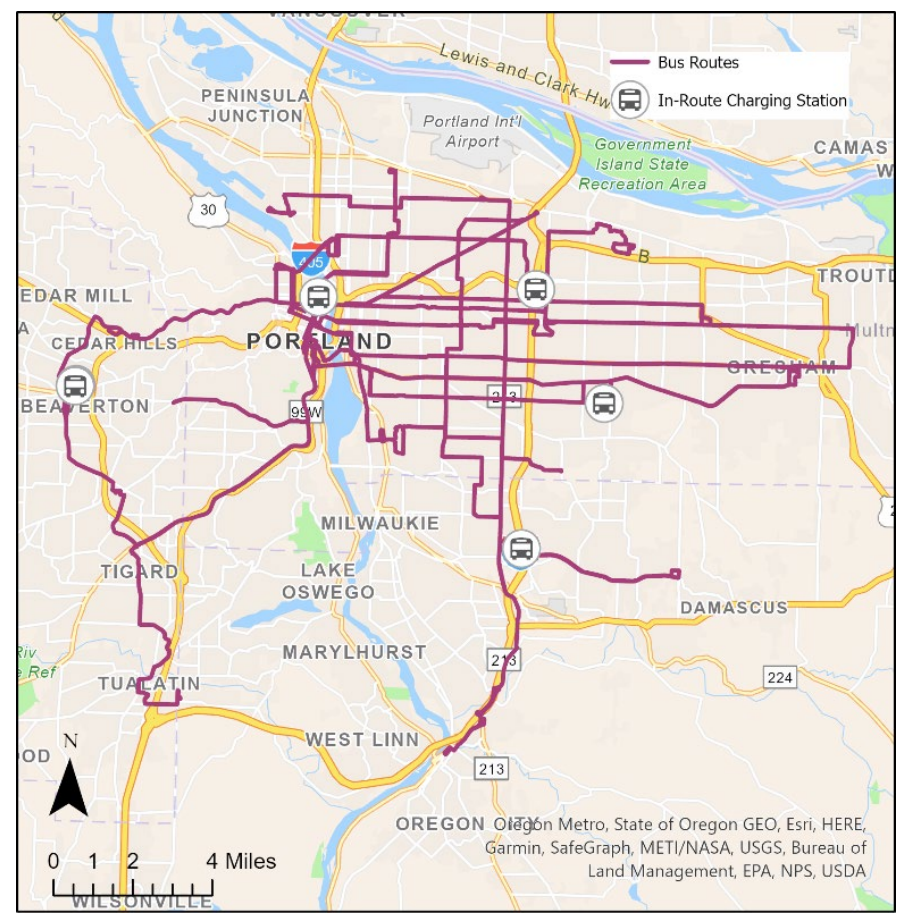

Figure 4.9: 50 BEB Replacements

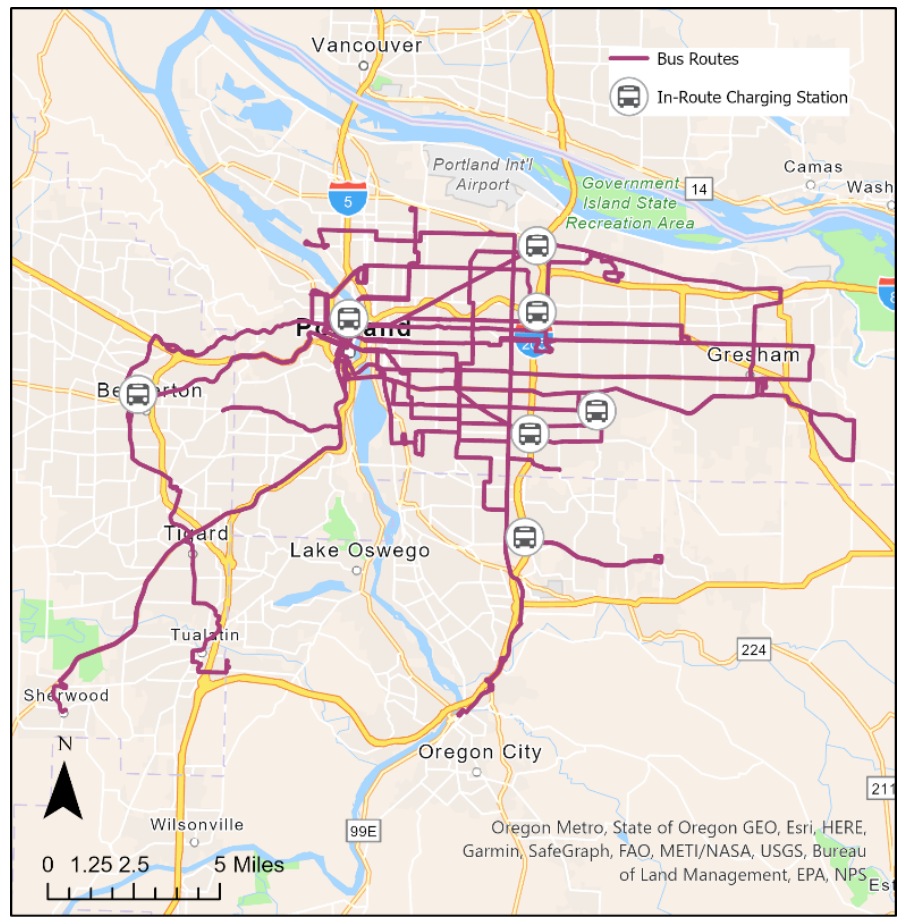

Figure 4.10: 70 BEB Replacements

Taking a closer look at the BOBEBD route selection in the 70 BEB replacement plan, the routes make intuitive sense by looking at their placement in juxtaposition with the TAZ map of income (Figure 4.10). Line 72 Killingsworth is a long route stretching over 17 miles, traveling in North and Northeast Portland along Killingsworth and parts of 
Alberta through the Humboldt, King, Vernon, Concordia, and Cully Neighborhoods. Route 72 then runs south along 82nd Ave through Roseway/Madison South, Montavilla, Mt. Scott, and the Lents neighborhoods, and into Johnson Creek. Many of these neighborhoods are well-known targets for equity policy. The anti-displacement and affordable housing preservation projects in neighborhoods like Cully and Lents have been ongoing in recent years.

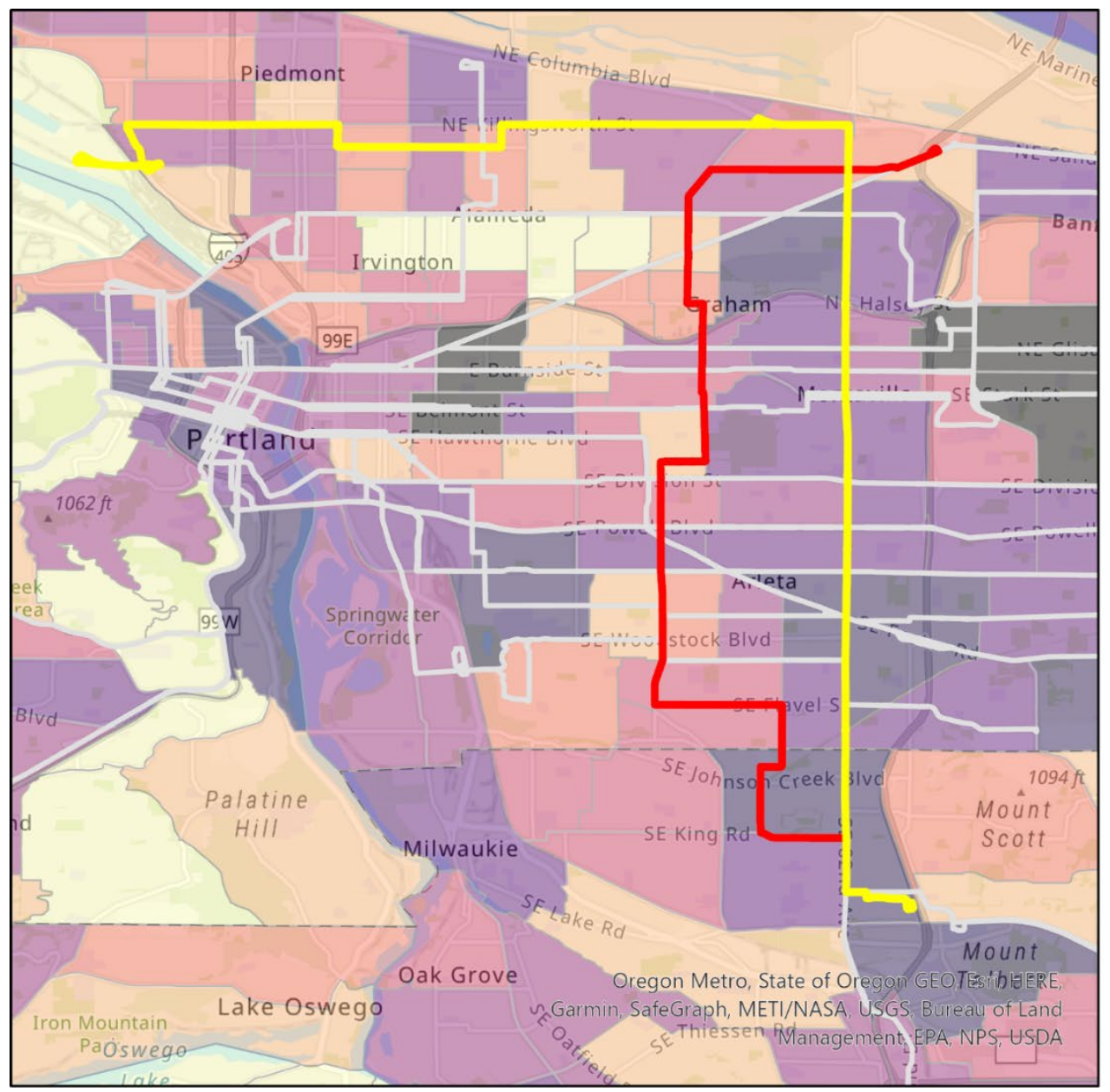

Low Income Households

ए 0 - 169

ए $167-300$

$301-416$

$417-575$

$576-745$

$746-969$

$970-1375$

$1376-2140$

Route Configuration for 70 BEB

71 - 60th Ave

72 - Killingsworth/82nd Ave

All Other Routes

Figure 4.11: Selected Lines 71 - 60th and 72 - Killingsworth/82 ${ }^{\text {nd }}$ Ave.

Looking at the Portland Bureau of Transportation (PBOT) Equity Matrix1 that considers race, income, and English proficiency in its calculation of an equity index, Figure 4.11 clear shows why Line 72 Killingsworth is one of the first routes selected in the model. This route is the dividing line that seemingly splits the city by race and income, with a clear distinction between the inner and outer East Portland area divided along 82nd Ave. 
There is a clear priority towards buses that serve long routes reaching out towards East Portland and Gresham, as would be expected by the distribution of low-income households. Of all the routes in this selection scenario, lines 2, $912,19,20,71$, and 72 receive the largest allocation of bus blocks. Each of these is a long bus route and contiguous with block groups containing a high proportion of low-income households.

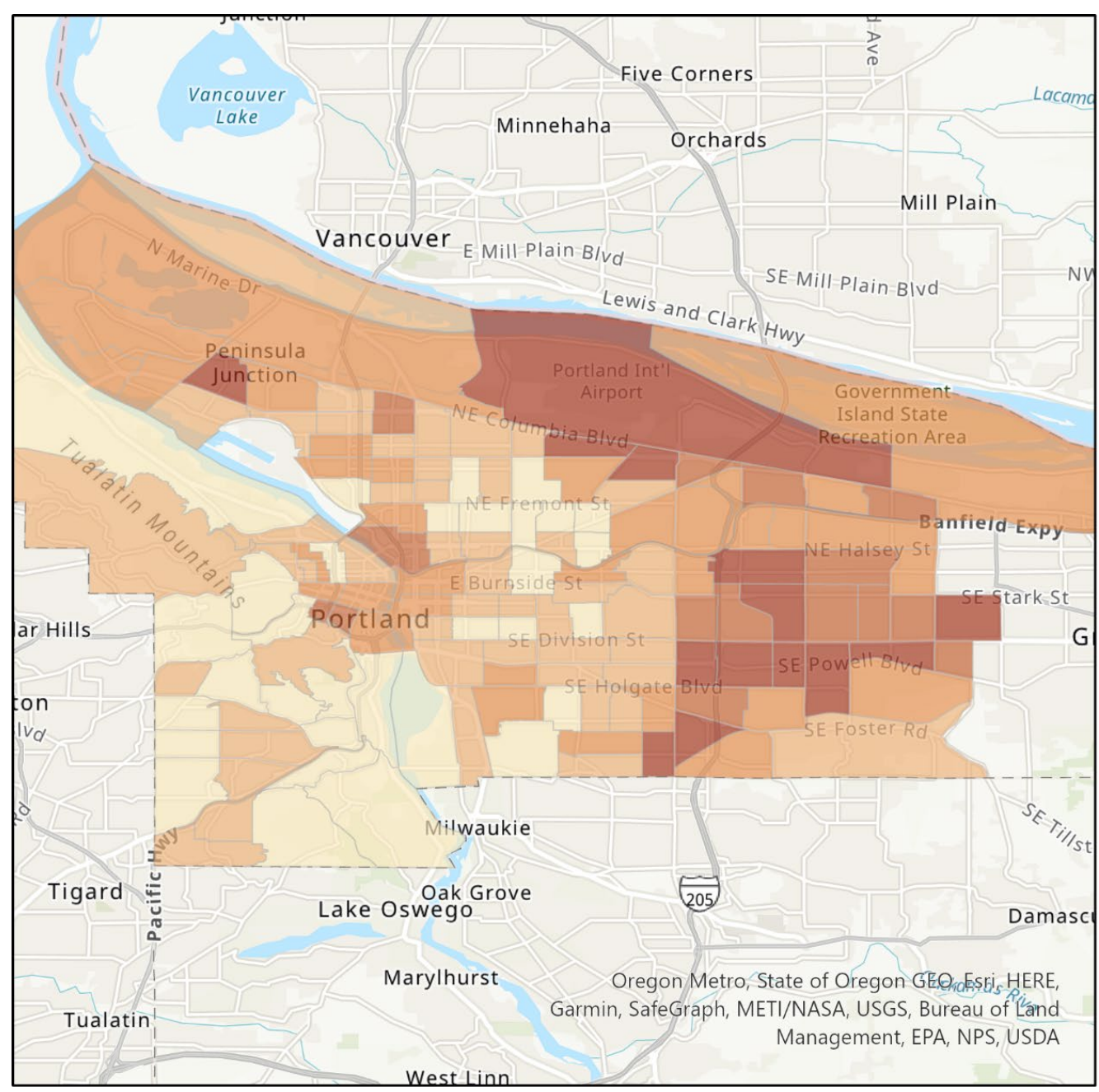

PBOT Equity Matrix

Combined Score

$2-3$

$3-6$

$6-8$

$8-9$

$9-10$

Figure 4.12: PBOT Equity Matrix 


\subsection{VISUALIZATION}

Based on the exploratory analysis of the data and results of the project, the visualization has been designed to include features such as multiple views, interactivity, and a combination of visuals and descriptive context.

\subsection{Visuals}

The visual components have been divided into three parts as shown in Figure 5.1. The first part is to demonstrate the data which is crucial for generating the final deployment plans. The second part is a street map which shows the actual deployment plan under a certain budget. The last part is a trade-off curve which allows users to choose from different budgets and to explore detailed information of the deployment plan. Note that all three parts are interconnective in nature, where the input from the user (e.g., third view budget plan) will also be reflected in the first and second views.

\section{Battery Electric Bus Deployment in the Greater} Salt Lake Region

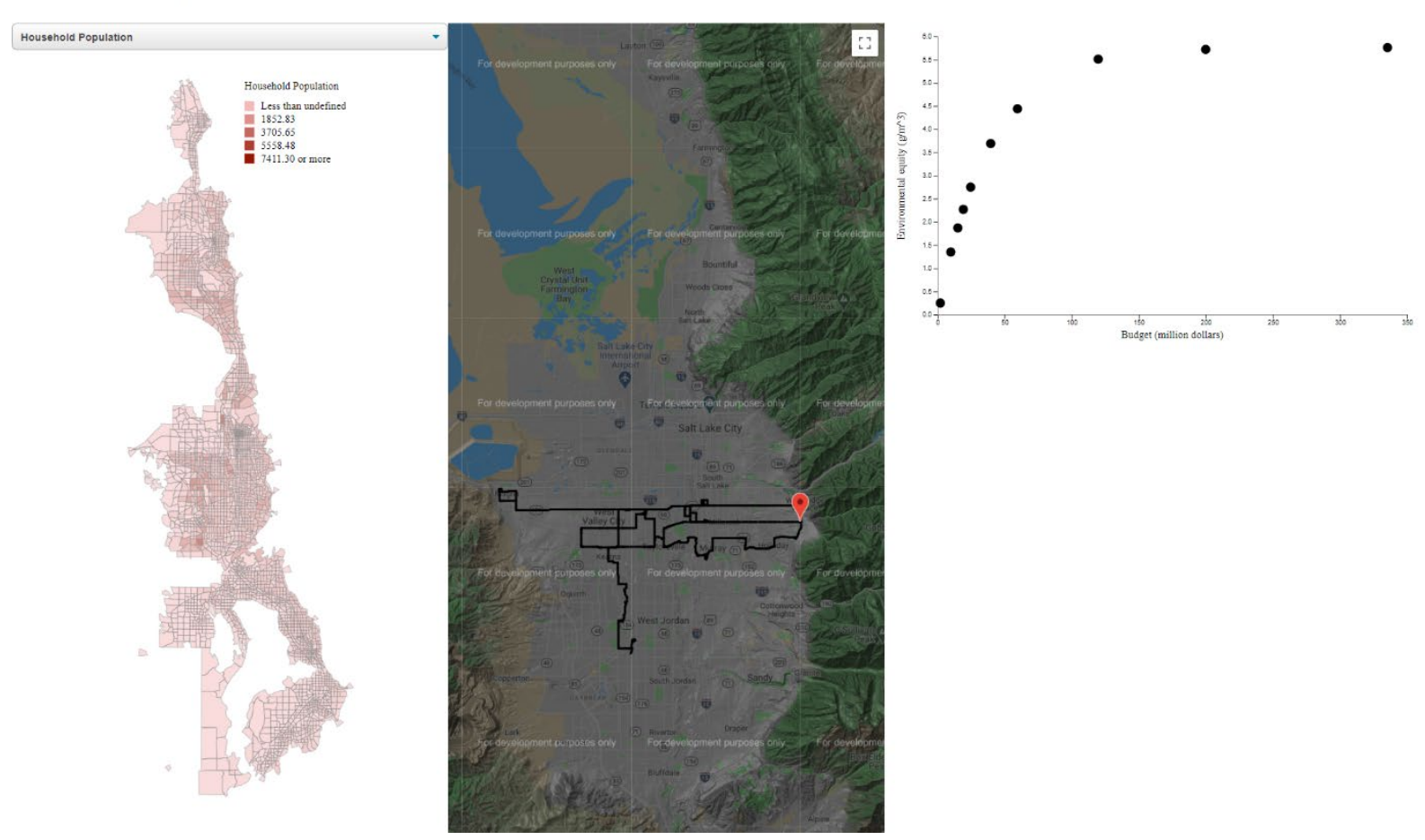

Figure 5.1: The Overall View of Visuals

\subsubsection{The First View}

The first view (Figure 5.2) is used to exhibit the basic data including the distribution of different income groups, pollution concentration, employment level, etc., which can be 
selected from the dropdown box at the top of the view. Upon selection, color-coded map distributions delineated by TAZ are presented. Information of individual TAZ can also be displayed when hovering cursor over.

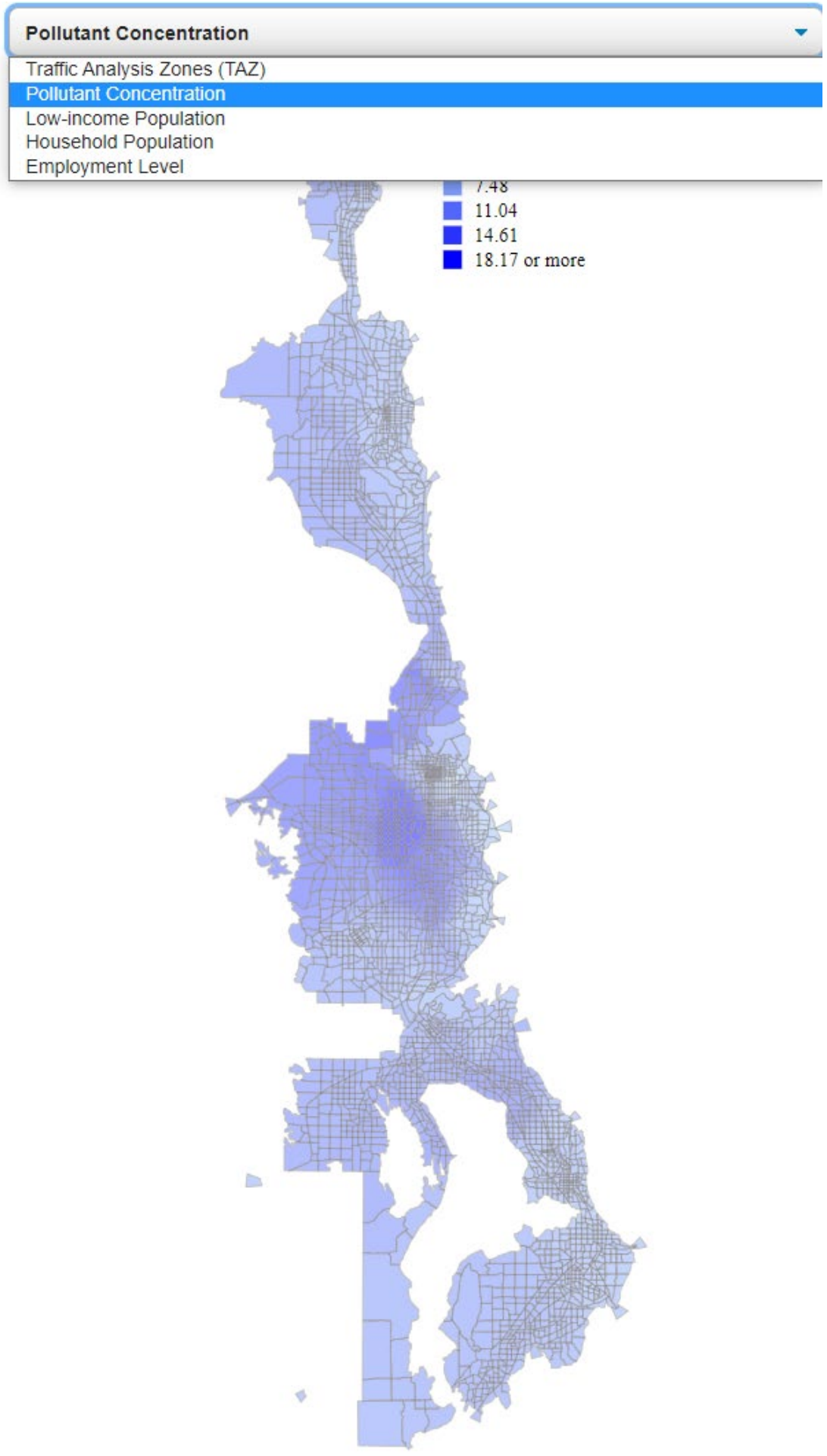

Figure 5.2: The First View 


\subsubsection{The Second View}

The second view (Figure 5.3) displays the actual deployment plan on a street map, which is scalable. The red mark represents the locations of on-route charging stations. The solid black lines are the bus routes where BEBs are traveling.

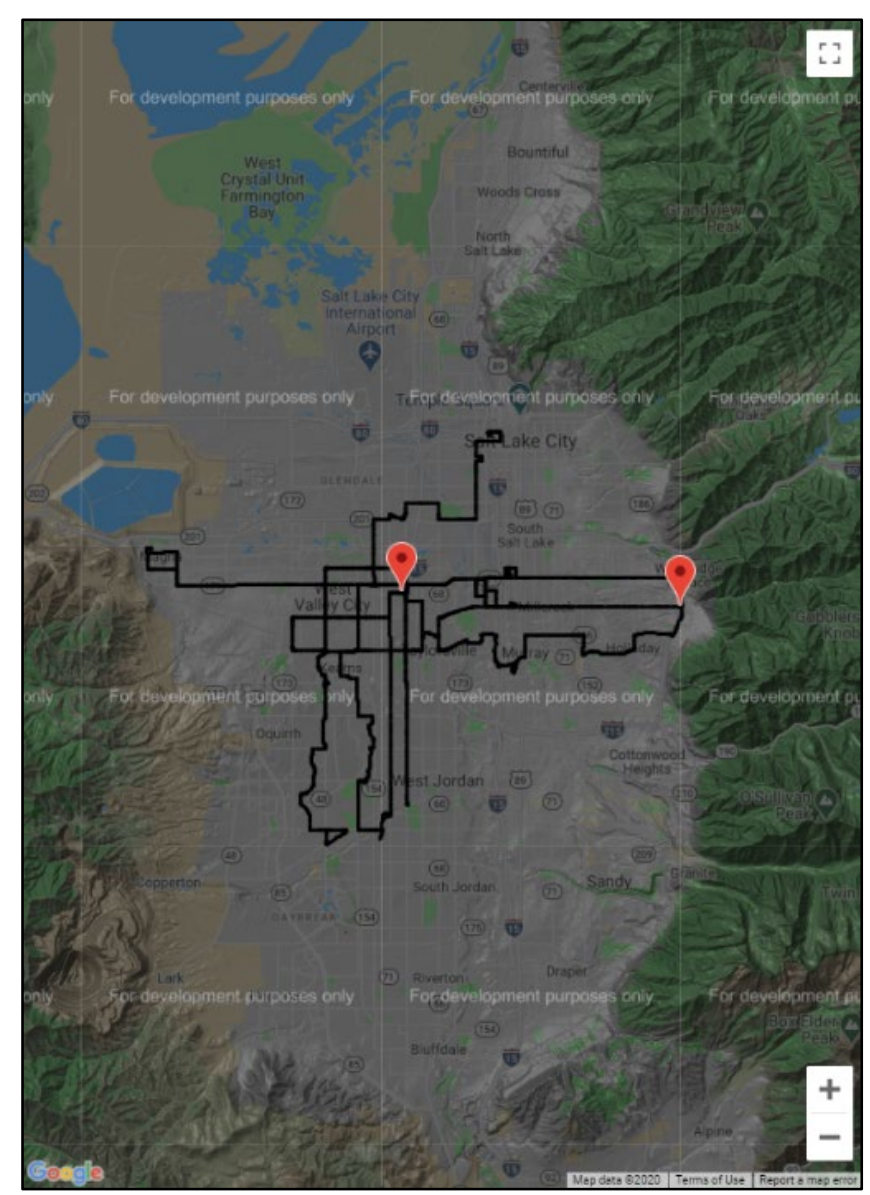

Figure 5.3: The Second View

\subsubsection{The Third View}

The third view (Figure 5.4) is the trade-off curve between budgets and environmental equity. Each point in the scatter plot is clickable and represents a different deployment plan which can be displayed in the second view (Figure 5.3). 


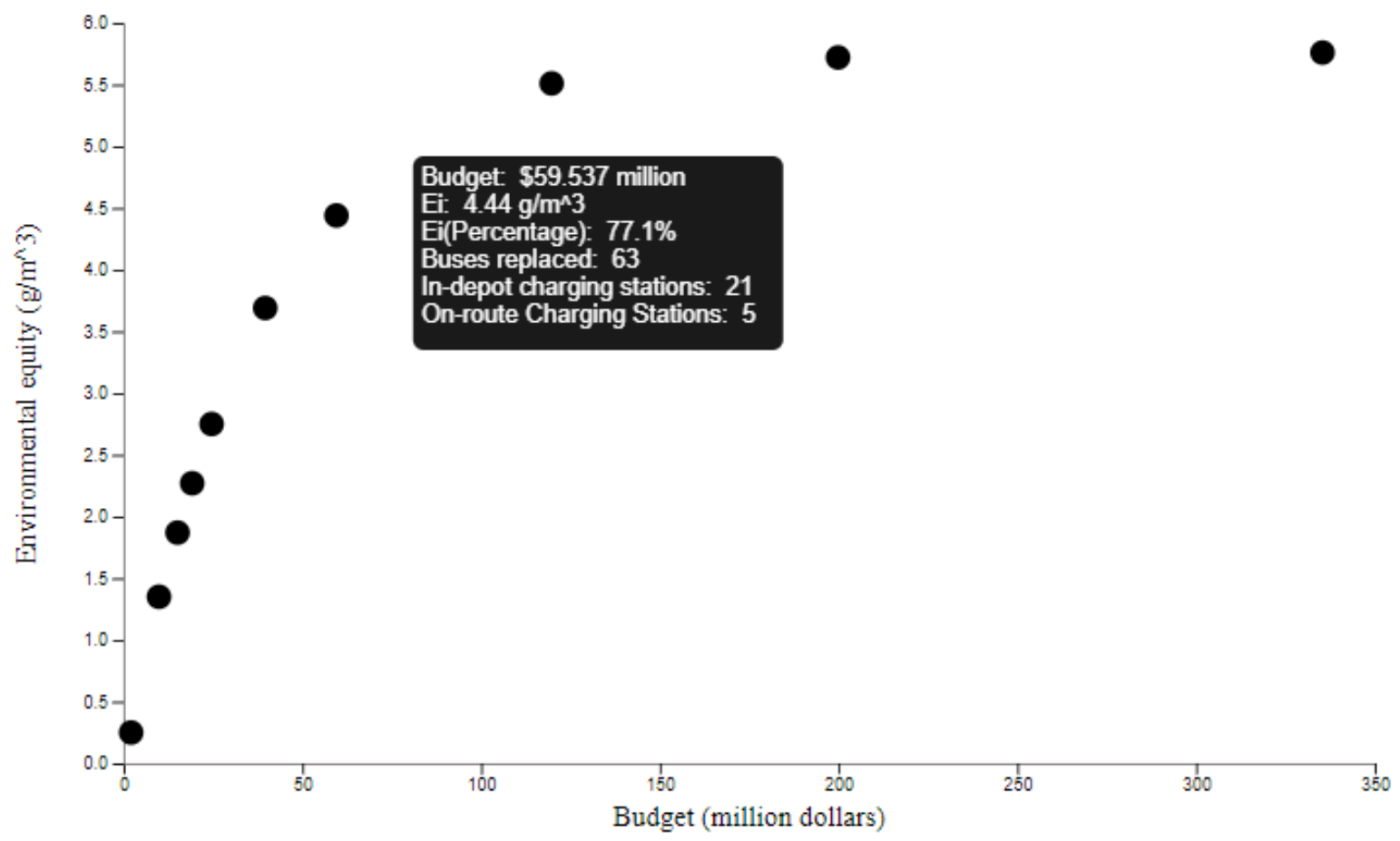

Figure 5.4: The Third View

\subsection{Descriptive Content}

To serve as the introduction of the project and complementary information of the visualizations, the descriptive content (Figure 5.5) is aligned below all the visual contents on the platform. 


\section{Dackgruma}

Environmental concerns due to fossil fuel consumption and emissions drive transportation industry to shift towards low-impact and sustainable energy sources. Public transit system, as an integral part of multimodal transportation ecosystem, has been supporting such shift by exploring the adoption of electric vehicles. In recent years, the advancement in Battery Electric Buses (BEBs) and their supporting infrastructure technology made them a viable replacement for diesel and Compressed Natural Gas (CNG) buses. Yet, it remains a challenge on how to optimally deploy the BEB system due to its unique spatio-temporal characteristics.

Utah Transit Authority (UTA), the public transportation provider throughout the Wasatch Front of Utah, has already begun the electrification of its bus fleet starting from 2016. 5 BEBs have been brought to service, among which three were used on route 2 and two served the University of Utah campus. After the successful initial release of BEBs, UTA has been working with the University of Utah to further study the possiblility of full electrification.

\section{Challenges}

While BEB and its supporting infrastructure have been commercialized and gradually adopted, how to optimally deploy the BEB system remains a challenge due to several unique spatio-temporal characteristics associated with the system itself. First, to support long daily operation time and high daily mileage, some BEBs would require both periodic on-route charging at bus terminals and overnight charging at bus garages. A careful planning for the optimal locations of on-route charging stations and overnight in-depot charging stations is necessary to efficiently serve the BEBs while keeping the cost minimal. Second, the space-time trajectories of BEBs should fit into current transit vehicle operation routes and schedules as much as possible, to enable smooth transition from traditional diesel or Compressed Natural Gas (CNG) buses to BEBs. The concern for potential interference with current operation routes and schedule would impede the acquisition of BEBs. It thus requires a sophisticated spatio-temporal analytical method to determine how to spatially and temporally integrate BEBs into current public transit system without interference with current operation routes and schedules.

\section{Related work}

Wei et al. (2018) developed an innovative spatio-temporal analytical framework to assist transit agencies in identifying the optimal deployment for the BEB system. Specifically, a spatio-temporal optimization model is developed to minimize the cost of replacing a certain number of diesel or CNG buses (part of the fleet) with BEBs, while in compliance with existing bus operation routes and schedules. The proposed model can be used to determine the optimal spatiotemporal allocation of the $\mathrm{BEBs}$, as well as the associated on-route charging stations and in-depot charging stations. The network data is obtained from UTA in year 2016 .

In addition, Yirong et al. futher enrich the strategical deployment framework of BEB by incorporating a second objective, environmental equity. The research develops a bi-objective spatio-temporal optimization model for the strategic deployment of BEB. The first objective is to minimize the cost of purchasing $\mathrm{BEB}$ and installing both on-route and in-depot charging stations while maintaining current bus schedules. The other objective is to maximize environmental equity by incorporating the disadvantaged population in the decision-making process. One main reason is that research on social vulnerability found that low socioeconomic status (SES) groups often experience a higher concentration of air pollutants, due to the low value of lands and the closeness to income-earning nnmortinnitis.

Figure 5.5: Descriptive Content

\subsection{CONCLUSION}

Among the several findings worthy of discussion, the first one is the shape of the tradeoff curve between budget and the environmental equity outcome. Figure 4.1 shows that the improvements regarding environmental equity work on a logarithmic scale as the budget continues to rise. It is due to the fact that $E_{i}$ varied significantly across buses, as illustrated in Figure 3.6. Some buses run routes that go through the most populated area multiple times a day while others might navigate through TAZs with very little 
population. Situations like this cause BOBEBD to almost always favor the buses on the densely populated routes. When $C_{x}=\$ 25$ million, all of the 26 buses chosen require both on-route and in-depot charging because they tend to operate longer routes and hours than those (114 buses) requiring only in-depot charging.

The BOBEBD is an illustration of how to formulate the deployment problem according to multiple needs and objectives set forth by the transit agencies given the unique spatiotemporal characteristics of BEBs. It can be extended to incorporate additional goals other than budget and environmental equity achieved, such as maximizing service area, fuel efficiency, the robustness of the system, etc. For example, robustness can be defined as the tolerable number of buses that can be allowed for malfunction with no or minimal impact on the current transit operation routes and schedules. In this case, buses that run fewer times a day, serve fewer routes, and stop longer at terminals might be the best candidates. Various goals can also be prioritized at different stages. For example, one goal at the early stage (e.g., $10 \%$ of fleet replaced with BEBs) can be to maximize the service coverage area to collect feedback from the community while the goal at the middle stage (e.g., $35 \%$ of fleet replaced with BEBs) can be to maximize environmental equity as demonstrated in this project. The flexibility of BOBEBD makes it possible for transit agencies to make planning-level decisions according to their shortterm and long-term goals along with specific requirements.

Also, there is plenty of room for improvement. At this point, we only considered the cost of purchasing BEBs and building charging stations. The difference in maintenance cost between BEBs and diesel or CNG buses and the residual value for specific buses could also be included in objective (2) if data is available. In addition, as we mentioned in the Data Source section, considering only the residents-based indicators (e.g., low-income residents) can potentially underestimate the number of served populations. Future work will focus on combining residents-based data with land use or other detailed human activities data to enhance estimation accuracy. Also, as discussed earlier, no partial charging is allowed and only terminals where buses dwell for more than 10 minutes are qualified as potential sites for building on-route charging stations. This feature is not in entire conformity with reality. As observed in the current BEB operation data in Salt Lake City, more than $50 \%$ of the time the BEB would get charged en route when it still had at least $30 \%$ of the total battery left. A closer assessment of the minimal amount of time for charging is needed for less conservative assumptions. With new technology emerging, such as wireless charging systems, improvements are necessary for BOBEBD as well.

The deployment of BEBs is a complex process that exerts huge impacts on transit systems, which requires enormous capital investment, thorough feasibility study, and careful planning. From the modeling perspective, the parameters of BEBs and the specifications of both on-route and in-depot charging stations (e.g., charging capability, charging time) determine which buses are feasible for replacement to maintain the same routes and schedules. The assumptions (e.g., no partial charging) we made could also greatly influence the allocation of on-route charging stations. This research contributes to the state-of-the-art BEB deployment by incorporating multiple objectives (cost and environmental equity). The joint usage of air pollution data, socio- 
demographic data, geographic information system (GIS), and optimization techniques offers a practical and strategic deployment that transit agencies can use. The BOBEBD enables transit agencies to balance capital investment and environmental equity and, with further adoption, to set forth various goals at different stages of deployment. This research lays the foundation for transit agencies to make multistage plans for deploying BEB using a flexible and easy-to-interpret optimization model.

\subsection{REFERENCES}

ACCESS Magazine, Is a Half-Mile Circle the Right Standard for TODs, accessed on: October 2020 [Online]. Available: https://www.accessmagazine.org/wpcontent/uploads/sites/7/2015/10/ls-a-Half-Mile-Circle-the-Right-Standard-for-TODs.pdf

Adrita Islam, Nicholas Lownes, "When to go electric? A parallel bus fleet replacement study," in Transp. Res. Part D, vol. 72, pp. 299-311, July 2019.

Alana Miller, Hye-Jin Kim, Jeffrey Robinson, Matthew Casale, Electric Buses, Clean Transportation for Healthier Neighborhoods and Clean Air, May 2018. Accessed on: April 2020. [Online]. Available:

https://uspirg.org/sites/pirg/files/reports/Electric\%20Buses\%20-\%20National\%20\%20May\%202018\%20web.pdf

Anders Nordelöf, Mia Romare, Johan Tivander, "Life cycle assessment of city buses powered by electricity, hydrogenated vegetable oil or diesel," in Transp. Res. Part D, vol. 75, pp. 211-222, October 2019.

Ángel Ibeas, Luigi dell'Olio, Borja Alonso, Olivia Sainz, "Optimizing bus stop spacing in urban areas," In Transp. Res. Part E, vol. 46, pp. 446-458, May 2010.

Anjum Hajat, Ana V. Diez-Roux, Sara D. Adar, Amy H. Auchincloss, Gina S. Lovasi, Marie S. O'Neill, Lianne Sheppard, Joel D. Kaufman. "Air Pollution and Individual and Neighborhood Socioeconomic Status: Evidence from the Multi-Ethnic Study of Atherosclerosis (MESA)," in Environ Health Perspect, vol. 121, no. 11-12, January 2013.

C. Liberto, G. Valenti, S. Orchi, M. Lelli, M. Nigro and M. Ferrara, "The Impact of Electric Mobility Scenarios in Large Urban Areas: The Rome Case Study," in IEEE Transactions on Intelligent Transportation Systems, vol. 19, no. 11, pp. 3540-3549, Nov. 2018.

Cohen, J. L., "Multiobjective programming and planning," New York: Academic Press, 1978.

Cressie, N., "The origins of kriging," in Math Geol 22, 239-252, April 1990. 
C. Yang, W. Lou, J. Yao and S. Xie, "On Charging Scheduling Optimization for a Wirelessly Charged Electric Bus System," in IEEE Transactions on Intelligent Transportation Systems, vol. 19, no. 6, pp. 1814-1826, June 2018.

Daniela Fecht, Paul Fischer, Léa Fortunato, Gerard Hoek, Kees de Hoogh, Marten Marra, Hanneke Kruize, Danielle Vienneau, Rob Beelen, Anna Hansell. "Associations between air pollution and socioeconomic characteristics, ethnicity and age profile of neighbourhoods in England and the Netherlands," in Environmental Pollution, vol. 198, pp. 201-210, March 2015.

Dennis Dreier, Semida Silveira, Dilip Khatiwada, Keiko V.O. Fonseca, Rafael Nieweglowski, Renan Schepanski, "Well-to-Wheel analysis of fossil energy use and greenhouse gas emissions for conventional, hybrid-electric and plug-in hybrid-electric city buses in the BRT system in Curitiba, Brazil," in Transp. Res. Part D, vol. 58, pp. 122-138, January 2018.

Elaine Croft McKenzie, Pablo L. Durango-Cohen, "Environmental life-cycle assessment of transit buses with alternative fuel technology," in Transp. Res. Part D, vol. 17, pp. 3947, December 2012.

Fang He, Di Wu, Yafeng Yin, Yongpei Guan, "Optimal deployment of public charging stations for plug-in hybrid electric vehicles," in Transportation Research Part B: Methodological, vol 47, pp.87-101, 0191-2615, January 2013.

Fang He, Yafeng Yin, Jing Zhou, "Deploying public charging stations for electric vehicles on urban road networks," in Transp. Res. Part C: Emerging Technologies, vol 60, pp.227-240, 0968-090X, November 2015.

Flamm, B. J., \& Rivasplata, C. R., "Public transit catchment areas: The curious case of cycle-transit users," in Transportation Research Record, vol. 2419, Issue 1, pp. 101108, January 2014.

Giovanni De Filippo, Vincenzo Marano, Ramteen Sioshansi, "Simulation of an electric transportation system at The Ohio State University," in Applied Energy, vol. 113, pp. 1686-1691, Juanuary 2014.

G. Tzeng, C. Lin, S. Opricovic, "Multi-criteria analysis of alternative-fuel buses for public transportation", in Energy Policy, vol. 33, no. 11, pp. 1373-1383, Jul. 2005.

Gurobi Optimization, Python Interface, accessed on: April 2020 [Online]. Available: https://www.gurobi.com/documentation/9.0/quickstart_mac/py_python_interface.html

Hajat, A., Hsia, C. \& O'Neill, M.S. "Socioeconomic Disparities and Air Pollution Exposure: a Global Review," in Curr Envir Health Rpt 2, 440-450, Septemper 2015.

Janecki R., Karoń G. "Concept of Smart Cities and Economic Model of Electric Buses Implementation," In Mikulski J. (eds) Telematics - Support for Transport. TST 2014. Communications in Computer and Information Science, vol. 471, pp. 100-109, 2014. 
Jing-Quan Li, "Battery-electric transit bus developments and operations: A review, " in Int. J. Sustainable Transp., 10:3, 157-169, January 2016.

Lajunen, A., "Energy consumption and cost-benefit analysis of hybrid and electric city buses" in Transp. Res. Part C, vol. 38, pp. 1-15, January 2014.

Luigi dell'Olio, Ángel Ibeas, F. Ruisánchez. "Optimizing bus-size and headway in transit networks," In Transportation, vol. 39, pp. 449-464, April 2011.

Maria Xylia, Semida Silveira, "The role of charging technologies in upscaling the use of electric buses in public transport: Experiences from demonstration projects," in Transport. Res. Part A, vol. 118, pp. 399-415, December 2018Michael Glotz-Richter, Hendrik Koch, "Electrification of Public Transport in Cities (Horizon 2020 ELIPTIC Project)," in Transport. Res. Procedia, vol. 14, pp. 2614-2619, April 2016.

Maria Xylia, Sylvain Leduc, Piera Patrizio, Florian Kraxner, Semida Silveira, "Locating charging infrastructure for electric buses in Stockholm," in Transp. Res. Part C, vol. 78, pp. 183-200, May 2017.

Mark Garrett, Brian Taylor, "Reconsidering Social Equity in Public Transit," in Berkeley Planning Journal, vol. 13, pp. 6-27, 1999.

Matthias Rupp, Christian Rieke, Nils Handschuh, Isabel Kuperjans, "Economic and ecological optimization of electric bus charging considering variable electricity prices and CO2eq intensities," in Transp. Res. Part D, vol. 81, 102293, April 2020.

Metro. (2019). TriMet's New Flyer Electric Buses Powered Entirely by Wind. Retrieved from Metro Magazine: https://www.metro-magazine.com/10031545/trimets-new-flyerelectric-buses-powered-entirely-by-wind

Michelle L. Bell, Keita Ebisu. "Environmental Inequality in Exposures to Airborne Particulate Matter Components in the United States," in Environ Health Perspect, vol. 120, no. 12, December 2012.

M. T. Sebastiani, R. Lüders and K. V. O. Fonseca, "Evaluating Electric Bus Operation for a Real-World BRT Public Transportation Using Simulation Optimization," in IEEE Transactions on Intelligent Transportation Systems, vol. 17, no. 10, pp. 2777-2786, Oct. 2016.

M. Kural, F. K. Tuncer, D. Memiş and M. N. Dai, "A Smart Mobility Platform for Electric Vehicles with Event Processing," in 2019 IEEE 5th World Forum on Internet of Things (WF-IoT), pp. 480-484, July 2019.

N.A. El-Taweel, A. Zidan and H. E. Z. Farag, "Novel Electric Bus Energy Consumption Model Based on Probabilistic Synthetic Speed Profile Integrated With HVAC," in IEEE Transacitons on Intelligent Transportation Systems, February 2020. 
Nan Qin, Azwirman Gusrialdi, R. Paul Brooker, Ali T-Raissi, "Numerical analysis of electric bus fast charging strategies for demand charge reduction," in Transp. Res. Part A, vol. 94, pp. 386-396, December 2016.

Pratt, Gregory C et al. "Traffic, air pollution, minority and socio-economic status: addressing inequities in exposure and risk." International journal of environmental research and public health vol. 12,5 5355-72. 19 May. 2015.

P. Sinhubera, W. Rohlfsa, D. Sauera, "Study on power and energy demand for sizing the energy storage systems for electrified local public transport buses", in Proc. IEEE Veh. Power Propulsion Conf., pp. 315-320, 2012.

PurpleAir, PurpelAir Map Data Layer, accessed on: April 2020 [Online]. Available: https://www.purpleair.com/map?opt=1/mAQl/a10/cC0\#11/40.7671/-111.8688

Ran Wei, Xiaoyue Liu, Yi Ou, S. Kiavash Fayyaz, "Optimizing the spatio-temporal deployment of battery electric bus system," in Journal of Transport Geography, vol. 68, pp. 160-168, April 2018

Samuel Pelletier, Ola Jabali, Jorge E. Mendoza, Gilbert Laporte, "The electric bus fleet transition problem," in Transp. Res. Part C, vol. 109, pp. 174-193, December 2019.

Spasovic, Lazar N., Maria P. Boile, and Athanassios K. Bladikas. "A methodological framework for optimizing bus transit service coverage," In 73rd Annual Meeting of the Transportation Research Board. 1993.

S. Kiavash Fayyaz, Xiaoyue Cathy Liu, Richard J. Porter, "Dynamic transit accessibility and transit gap causality analysis," in Journal of Transport Geography, vol. 59, pp 2739, Feburary 2017.

Tao Liu, Avishai (Avi) Ceder, "Battery-electric transit vehicle scheduling with optimal number of stationary chargers," in Transp. Res. Part C, vol. 114, pp. 118-139, May 2020.

Toh CK, Sanguesa JA, Cano JC, Martinez FJ. "Advances in smart roads for future smart cities," In Proc Math Phys Eng Sci, vol. 476, Januaty 2020.

Trimet. (2018). TrimeNon-Diesel Bus Plan.

TriMet. (2019). TriMet Electric Bus Plan. Retrieved from http://media.oregonlive.com/commuting/other/TriMet\%20Electric\%20Bus\%20Plan\%209. 12.18.pdf 
TriMet. (2020). Proposed Fiscal Year Budget. Retrieved from https://trimet.org/budget/\#: :text=The\%20FY2021\%20Adopted\%20Budget\%20totals,fun ding $\% 20$ of $\% 20$ approximately $\% 20 \% 24126 \% 20$ million.

United States Environmental Protection Agency, Utah Nonattainment/Maintenance Status for Each County by Year for All Criteria Pollutants, accesed on: April 2020 [Online]. Available: https://www3.epa.gov/airquality/greenbook/anayo_ut.html

UTA, First/Last Mile Strategies Study, accessed on: October 2020 [Online]. Available: https://www.rideuta.com/-/media/Files/About-UTA/TigerVIII/UTAFirst_LastMileFINALCOMP1.ashx?la=en

X. Wang, C. Yuen, N. U. Hassan, N. An and W. Wu, "Electric Vehicle Charging Station Placement for Urban Public Bus Systems," in IEEE Transactions on Intelligent Transportation Systems, vol. 18, no. 1, pp. 128-139, Jan. 2017.

Yusheng Wang, Yongxi Huang, Jiuping Xu, Nicole Barclay, "Optimal recharging scheduling for urban electric buses: A case study in Davis, " in Transp. Res. Part E, vol. 100, pp. 115-132, April 2017.

Y. Zhou, X. C. Liu, R. Wei and A. Golub, "Bi-Objective Optimization for Battery Electric Bus Deployment Considering Cost and Environmental Equity," in IEEE Transactions on Intelligent Transportation Systems, doi: 10.1109/TITS.2020.3043687. 